\title{
FIBER STRUCTURE AND LOCAL COORDINATES FOR THE TEICHMÜLLER SPACE OF A BORDERED RIEMANN SURFACE
}

\author{
DAVID RADNELL AND ERIC SCHIPPERS
}

\begin{abstract}
We show that the infinite-dimensional Teichmüller space of a Riemann surface whose boundary consists of $n$ closed curves is a holomorphic fiber space over the Teichmüller space of an $n$-punctured surface. Each fiber is a complex Banach manifold modeled on a two-dimensional extension of the universal Teichmüller space. The local model of the fiber, together with the coordinates from internal Schiffer variation, provides new holomorphic local coordinates for the infinite-dimensional Teichmüller space.
\end{abstract}

\section{INTRODUCTION}

1.1. Statement of results. Let $\mathbb{D}=\{z \in \mathbb{C}:|z|<1\}, \mathbb{D}_{0}=\mathbb{D} \backslash\{0\}$, and $\overline{\mathbb{D}}_{0}=\{z \in \mathbb{C}: 0<|z| \leq 1\}$. In the following, the term "bordered" Riemann surface refers to a Riemann surface with boundary in the standard sense (see e.g. [1]).

Definition 1.1. We say that $\Sigma^{B}$ is a bordered Riemann surface of type $(g, n)$ if (1) its boundary consists of $n$ ordered closed curves homeomorphic to $S^{1}$ and (2) it is biholomorphically equivalent to a compact Riemann surface of genus $g$ with $n$ simply-connected non-overlapping regions, biholomorphic to $\mathbb{D}$, removed. We say that $\Sigma^{P}$ is a punctured Riemann surface of type $(g, n)$ if it is biholomorphically equivalent to a compact Riemann surface with $n$ points $p_{1}, \ldots, p_{n}$ removed.

Note that it is assumed that bordered Riemann surfaces have no punctures, and that the boundary components of punctured Riemann surfaces consist only of single points. We will denote bordered Riemann surfaces of type $(g, n)$ with a superscript $B$ and punctured Riemann surfaces with a superscript $P$.

Remark 1.2. One can also view a punctured Riemann surface $\Sigma^{P}$ as a compact Riemann surface with $n$ distinguished points.

Remark 1.3. Throughout the paper, we consider only Riemann surfaces with no non-trivial automorphisms that are homotopic to the identity. That is, we do not consider the special cases where $2 g-2+n \leq 0$. The important special case when $\Sigma^{B}$ is an annulus is the topic of the subsequent article [15] by the authors.

Received by the editors June 17, 2009.

2010 Mathematics Subject Classification. Primary 30F60, 58B12; Secondary 81T40.

Key words and phrases. Teichmüller spaces, quasiconformal mappings, sewing, rigged Riemann surfaces, conformal field theory.

(C)2010 American Mathematical Society Reverts to public domain 28 years from publication 
Given a bordered Riemann surface $\Sigma^{B}$ of type $(g, n)$, we can obtain a punctured Riemann surface $\Sigma^{P}$ in the following way. For details, see [13, Section 3]. Denote the boundary components by $\partial_{i} \Sigma^{B}$. Let $\tau=\left(\tau_{1}, \ldots, \tau_{n}\right)$ where each $\tau_{i}: \partial \mathbb{D} \rightarrow \partial_{i} \Sigma^{B}$ is a fixed quasisymmetric mapping. For the purposes of this paper, we say that such a $\tau_{i}$ is quasisymmetric if $\tau_{i}$ extends to a quasiconformal map of $\{z: 1<|z|<r\}$ into a doubly-connected neighborhood of $\partial_{i} \Sigma^{B}$ [13, Definition 2.12]. The boundary parametrizations $\tau$ are referred to as a "rigging". We sew on $n$ copies of the punctured unit disc $\overline{\mathbb{D}}_{0}$, denoted $\overline{\mathbb{D}}_{0, i}$, to $\Sigma^{B}$ as follows. Consider the disjoint union of $\Sigma^{B}$ and $\overline{\mathbb{D}}_{0,1} \sqcup \cdots \sqcup \overline{\mathbb{D}}_{0, n}$. Identifying boundary points using $\tau$, the result is a compact surface $\Sigma^{P}$ with $n$ punctures $p_{i}$ corresponding to each puncture in $\overline{\mathbb{D}}_{0}$. That is, let

$$
\Sigma^{P}=\left(\Sigma^{B} \sqcup \overline{\mathbb{D}}_{0,1} \sqcup \cdots \sqcup \overline{\mathbb{D}}_{0, n}\right) / \sim
$$

where $p \sim q$ if $p \in \partial_{i} \Sigma^{B}$ and $q \in \partial \mathbb{D}_{i}$, and $p=\tau_{i}(q)$. By [13, Theorem 3.3] $\Sigma^{P}$ has a unique complex structure which is compatible with that of both $\Sigma^{B}$ and $\overline{\mathbb{D}}_{0, i}$ for all $i$. (Note that the punctures of $\Sigma^{P}$ are also ordered.) If $\Sigma^{P}$ is obtained from $\Sigma^{B}$

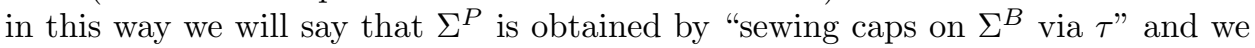
write

$$
\Sigma^{P}=\Sigma^{B} \#_{\tau} \overline{\mathbb{D}}_{0}^{n}
$$

The parametrizations $\tau_{i}$ can be extended to maps $\tilde{\tau}_{i}: \overline{\mathbb{D}}_{0} \rightarrow \Sigma^{P}$ to the caps of $\Sigma^{P}$ by

$$
\tilde{\tau}_{i}(x)= \begin{cases}\tau_{i}(x), & \text { for } x \in \partial \mathbb{D}, \\ x, & \text { for } x \in \mathbb{D}_{0} .\end{cases}
$$

These maps $\tilde{\tau}_{i}$ have quasiconformal extensions to a neighborhood of $\overline{\mathbb{D}}_{0}$.

The aim of this paper is to show that the infinite-dimensional Teichmüller space $T\left(\Sigma^{B}\right)$ of $\Sigma^{B}$ is a holomorphic fiber space over the finite-dimensional Teichmüller space $T\left(\Sigma^{P}\right)$ of $\Sigma^{P}$. The fibers can be explicitly described as follows.

Definition 1.4 ( of $\left.\mathcal{O}_{q c}\left(\Sigma_{*}^{P}\right)\right)$. Let $\Sigma_{*}^{P}$ be a compact Riemann surface of type $(g, n)$ with distinguished points $p_{1}, \ldots, p_{n}$. Define $\mathcal{O}_{\mathrm{qc}}\left(\Sigma_{*}^{P}\right)$ to be the set of $n$-tuples $\left(\phi_{1}, \ldots, \phi_{n}\right)$ where $\phi_{i}: \mathbb{D} \rightarrow \Sigma_{*}^{P}$ are maps with the following properties:

(1) $\phi_{i}(0)=p_{i}$,

(2) $\phi_{i}$ is conformal on $\mathbb{D}$,

(3) $\phi_{i}$ has a quasiconformal extension to a neighborhood of $\overline{\mathbb{D}}$,

(4) $\phi_{i}(\overline{\mathbb{D}}) \cap \phi_{j}(\overline{\mathbb{D}})=\emptyset$ whenever $i \neq j$.

It is convenient to single out the following case.

Definition 1.5. Let $\mathcal{O}_{\mathrm{qc}}$ denote the set of holomorphic univalent functions $f$ : $\mathbb{D} \rightarrow \mathbb{C}$ with quasiconformal extensions to $\mathbb{C}$ satisfying the normalization $f(0)=0$.

In 14 it was shown that $\mathcal{O}_{\mathrm{qc}}$ possesses a natural complex structure related to that of the universal Teichmüller space, and that $\mathcal{O}_{\mathrm{qc}}\left(\Sigma_{*}^{P}\right)$ is a complex Banach manifold which is locally modeled on $\mathcal{O}_{\mathrm{qc}}^{n}=\mathcal{O}_{\mathrm{qc}} \times \cdots \times \mathcal{O}_{\mathrm{qc}}$.

Let $\Sigma^{P}$ be the punctured Riemann surface obtained from $\Sigma^{B}$ by sewing on caps via $\tau$, and let $\Sigma_{*}^{P}$ be a marked Riemann surface representing an element of the Teichmüller space $T\left(\Sigma^{P}\right)$ of $\Sigma^{P}$. We show that the fiber in $T\left(\Sigma^{B}\right)$ over this element of $T\left(\Sigma^{P}\right)$, modulo the action of Dehn twists around curves homotopic to boundary curves, is in a natural one-to-one correspondence with $\mathcal{O}_{\mathrm{qc}}\left(\Sigma_{*}^{P}\right)$. 
We summarize the main results. Precise definitions and careful statements of the theorems can be found in subsequent sections.

\section{Summary of results.}

(1) If $\Sigma^{B}$ is a bordered Riemann surface of type $(g, n)$ (with $2 g-2+n>0$ ), then the Teichmüller space $T\left(\Sigma^{B}\right)$ is a complex fiber space over $T\left(\Sigma^{P}\right)$.

(2) Let $F_{B}\left(\left[\Sigma^{P}, f, \Sigma_{*}^{P}\right]\right)$ be the fiber over $\left[\Sigma^{P}, f, \Sigma_{*}^{P}\right] \in T\left(\Sigma^{P}\right)$. Let DB be the subgroup of the mapping class group of $\Sigma^{B}$ corresponding to Dehn twists around curves homotopic to boundary curves. Then $F_{B}\left(\left[\Sigma^{P}, f, \Sigma_{*}^{P}\right]\right) / \mathrm{DB}$ is biholomorphic to $\mathcal{O}_{\mathrm{qc}}\left(\Sigma_{*}^{P}\right)$. In particular, $F_{B}\left(\left[\Sigma^{P}, f, \Sigma_{*}^{P}\right]\right)$ is locally biholomorphic to the function space $\mathcal{O}_{\text {qc }}^{n}$.

(3) Schiffer variation coordinates on $T\left(\Sigma^{P}\right)$ together with open subsets of $\mathcal{O}_{\mathrm{qc}}\left(\Sigma_{*}^{P}\right)$ give local holomorphic coordinates for $T\left(\Sigma^{B}\right)$.

Some recent results are a key part of the formulation and proofs of these theorems. First is the authors' construction in [13] of a complex structure on the "rigged moduli space" and its explicit relation to the Teichmüller space $T\left(\Sigma^{B}\right)$. This construction uses ideas from two-dimensional conformal field theory in an essential way. A further crucial result from [13] is that the operation of sewing two Riemann surfaces with a quasisymmetric boundary identification is holomorphic in Teichmüller space. The complex structure on $\mathcal{O}_{\mathrm{qc}}\left(\Sigma_{*}^{P}\right)$ was constructed in [14].

A further tool is Gardiner's construction of coordinates on the Teichmüller space of a punctured surface of finite type using Schiffer variation 3. We use his method in order to construct a section of the projection of $T\left(\Sigma^{B}\right)$ onto $T\left(\Sigma^{P}\right)$. In the first author's thesis 12, Schiffer variation was used in an analogous way, although for the different purpose of defining a complex structure on the analytically rigged Teichmüller space.

The results above are an application of Segal's [16] formulation of conformal field theory to Teichmüller theory.

\section{Fiber structure of $T\left(\Sigma^{B}\right)$}

2.1. Teichmüller spaces. We now define the relevant Teichmüller spaces, to fix notation.

Let $\Sigma^{B}$ be a bordered Riemann surface of type $(g, n)$. Consider the set of triples $\left\{\left(\Sigma^{B}, f_{1}, \Sigma_{1}^{B}\right)\right\}$ where $\Sigma^{B}$ is a fixed Riemann surface, $\Sigma_{1}^{B}$ is another Riemann surface and $f_{1}: \Sigma^{B} \rightarrow \Sigma_{1}^{B}$ is a quasiconformal map (the "marking"). We say that $\left(\Sigma^{B}, f_{1}, \Sigma_{1}^{B}\right) \sim\left(\Sigma^{B}, f_{2}, \Sigma_{2}^{B}\right)$ if there exists a biholomorphism $\sigma: \Sigma_{1}^{B} \rightarrow \Sigma_{2}^{B}$ such that $f_{2}^{-1} \circ \sigma \circ f_{1}$ is homotopic to the identity "rel boundary", i.e. in such a way that the restriction of $f_{2}^{-1} \circ \sigma \circ f_{1}$ to the boundary is the identity throughout the homotopy.

Definition 2.1. The Teichmüller space of $\Sigma^{B}$ is

$$
T\left(\Sigma^{B}\right)=\left\{\left(\Sigma^{B}, f_{1}, \Sigma_{1}^{B}\right)\right\} / \sim
$$

We denote the equivalence classes by $\left[\Sigma^{B}, f_{1}, \Sigma_{1}^{B}\right]$. The case $T\left(\mathbb{D}^{*}\right)$, where $\mathbb{D}^{*}=$ $\{z:|z|>1\} \cup\{\infty\}$, is the universal Teichmüller space.

It is well known that $T\left(\Sigma^{B}\right)$ is a complex Banach manifold with complex structure compatible with the space $L_{-1,1}^{\infty}\left(\Sigma^{B}\right)$ of Beltrami differentials $\mu$ on $\Sigma^{B}$ in 
the following sense. Let $L_{-1,1}^{\infty}\left(\Sigma^{B}\right)_{1}$ denote the unit ball in the space of Beltrami differentials. Let

$$
\Phi: L_{-1,1}^{\infty}\left(\Sigma^{B}\right)_{1} \rightarrow T\left(\Sigma^{B}\right)
$$

be the fundamental projection, given by taking a Beltrami differential $\mu$ to $\left[\Sigma^{B}, f^{\mu}, \Sigma^{\mu}\right]$ where $f^{\mu}$ is a quasiconformal map of dilatation $\mu$. $\Phi$ is well defined.

Theorem 2.2 (see [8] and [11]). $\Phi$ is holomorphic and possesses local holomorphic sections.

Similarly, we can define the Teichmüller space of a punctured Riemann surface. Let $\Sigma^{P}$ be a punctured Riemann surface of type $(g, n)$. Consider the set of triples $\left(\Sigma^{P}, f_{1}, \Sigma_{1}^{P}\right)$ where $\Sigma^{P}$ is a fixed punctured Riemann surface, and $f_{1}: \Sigma^{P} \rightarrow \Sigma_{1}^{P}$ is a quasiconformal map onto the Riemann surface $\Sigma_{1}^{P}$. We say that $\left(\Sigma^{P}, f_{1}, \Sigma_{1}^{P}\right) \sim$ $\left(\Sigma^{P}, f_{2}, \Sigma_{2}^{P}\right)$ if there is a biholomorphism $\sigma: \Sigma_{1}^{P} \rightarrow \Sigma_{2}^{P}$ such that $f_{2}^{-1} \circ \sigma \circ f_{1}$ is homotopic to the identity.

Definition 2.3. The Teichmüller space of $\Sigma^{P}$ is defined by

$$
T\left(\Sigma^{P}\right)=\left\{\left(\Sigma^{P}, f_{1}, \Sigma_{1}^{P}\right)\right\} / \sim .
$$

We denote the equivalence classes by $\left[\Sigma^{P}, f_{1}, \Sigma_{1}^{P}\right]$. The Teichmüller space $T\left(\Sigma^{P}\right)$ is a complex manifold of dimension $3 g-3+n$.

Remark 2.4. Observing that quasiconformal and holomorphic maps must extend to the punctures, one obtains an alternate description of punctured Riemann surfaces and their Teichmüller spaces. One regards a punctured Riemann surface as a compact surface with distinguished points, and the definition of $T\left(\Sigma^{P}\right)$ is altered to require that the quasiconformal mappings take punctures to punctures, and the homotopy is "rel punctures"; that is, it preserves the punctures throughout.

Remark 2.5. Of course, the Teichmüller spaces of punctured and bordered Riemann surfaces are special cases of the general definition of the Teichmüller space of any Riemann surface covered by the disc.

As in [13, section 2.1] we introduce a certain subgroup of the mapping class group. The pure mapping class group of $\Sigma^{B}$ is the group of homotopy classes of quasiconformal self-mappings of $\Sigma^{B}$ which preserve the ordering of the boundary components. Let $\operatorname{PModI}\left(\Sigma^{B}\right)$ be the subgroup of the mapping class group consisting of equivalence classes of mappings that are the identity on the boundary $\partial \Sigma^{B}$. The group $\operatorname{PModI}\left(\Sigma^{B}\right)$ is finitely generated by Dehn twists.

Definition 2.6. Let $\mathrm{DB}\left(\Sigma^{B}\right)$ be the subgroup of $\operatorname{PModI}\left(\Sigma^{B}\right)$ generated by the equivalence classes of mappings which are Dehn twists around curves that are homotopic to boundary curves. We write DB when the surface is clear from context.

If $\Sigma^{B}$ is not an annulus or a disk, then $\operatorname{DB}\left(\Sigma^{B}\right)$ is isomorphic to $\mathbb{Z}^{n}$ and is in the center of $\operatorname{PModI}\left(\Sigma^{B}\right)$.

The mapping class group acts on $T\left(\Sigma^{B}\right)$ by $[\rho] \cdot\left[\Sigma^{B}, f, \Sigma_{1}^{B}\right]=\left[\Sigma^{B}, f \circ \rho, \Sigma_{1}^{B}\right]$.

Proposition 2.7 (see [13, Lemmas 5.1 and 5.2]). The group $\operatorname{PModI}\left(\Sigma^{B}\right)$, and hence its subgroup $\mathrm{DB}\left(\Sigma^{B}\right)$, acts properly discontinuously and fixed-point freely by biholomorphisms on $T\left(\Sigma^{B}\right)$. 
2.2. Description of the fibration. In this section we describe the fibers. This requires the use of a "rigged Teichmüller space", a concept motivated by conformal field theory, which we will define in the next paragraph. Details and proofs of the statements in this section were given in [13.

Definition 2.8. Let $\Sigma^{P}$ be a type $(g, n)$ punctured Riemann surface. Consider the set of quadruples $\left(\Sigma^{P}, f_{1}, \Sigma_{1}^{P}, \phi\right)$ where $\Sigma_{1}^{P}$ is a Riemann surface, $f_{1}: \Sigma^{P} \rightarrow \Sigma_{1}^{P}$ is a quasiconformal marking map as in the definition of $T\left(\Sigma^{P}\right)$, and $\phi \in \mathcal{O}_{\mathrm{qc}}\left(\Sigma_{1}^{P}\right)$. Define the equivalence relation $\left(\Sigma^{P}, f_{1}, \Sigma_{1}^{P}, \phi_{1}\right) \sim\left(\Sigma^{P}, f_{2}, \Sigma_{2}^{P}, \phi_{2}\right)$ to hold if and only if there exists a biholomorphism $\sigma: \Sigma_{1}^{P} \rightarrow \Sigma_{2}^{P}$ which preserves the punctures and their ordering such that $\sigma \circ \phi_{1}=\phi_{2}$ on $\partial \mathbb{D}$ (and hence on $\mathbb{D}$ ) and $f_{2}^{-1} \circ \sigma \circ f_{1}$ is homotopic to the identity. We then define

$$
\widetilde{T}^{P}\left(\Sigma^{P}\right)=\left\{\left(\Sigma^{P}, f_{1}, \Sigma_{1}^{P}, \phi\right)\right\} / \sim .
$$

The rigged Teichmüller space $\widetilde{T}^{P}\left(\Sigma^{P}\right)$ possesses a complex structure obtained in the following way. First, we have a bijection

$$
\widetilde{T}^{P}\left(\Sigma^{P}\right) \cong T\left(\Sigma^{B}\right) / \mathrm{DB}
$$

where DB and its action are defined in Section 2.1. By Proposition 2.7. $\widetilde{T}^{P}\left(\Sigma^{P}\right)$ inherits a complex structure from $T\left(\Sigma^{B}\right)$, and the bijection is in fact a biholomorphism [13, Theorem 5.7 parts (3) and (4)].

Next define the map $\mathcal{P}: T\left(\Sigma^{B}\right) \rightarrow \widetilde{T}^{P}\left(\Sigma^{P}\right)$ from [13, Section 5.4] in the following way (note that there, the map is denoted $P_{\mathrm{DB}}$ ). Fix a base parametrization $\tau$ : $\partial \mathbb{D}^{n} \rightarrow \partial \Sigma^{B}$ and a base Riemann surface $\Sigma^{P}=\Sigma^{B} \#_{\tau} \overline{\mathbb{D}}_{0}^{n}$. Given $\left[\Sigma^{B}, h, \Sigma_{1}^{B}\right] \in$ $T\left(\Sigma^{B}\right)$, let $\Sigma_{1}^{P}=\Sigma_{1}^{B} \#_{h \circ \tau} \overline{\mathbb{D}}_{0}^{n}$, and define $\tilde{h}: \Sigma^{P} \rightarrow \Sigma_{1}^{P}$ by

$$
\tilde{h}= \begin{cases}h, & \text { on } \Sigma^{B}, \\ \mathrm{id}, & \text { on } \mathbb{D}_{0}^{n} .\end{cases}
$$

Define $\tilde{\tau}: \overline{\mathbb{D}}_{0}^{n} \rightarrow \Sigma^{P}$ by (1.1), and set

$$
\mathcal{P}\left(\left[\Sigma^{B}, h, \Sigma_{1}^{B}\right]\right)=\left[\Sigma^{P}, \tilde{h}, \Sigma_{1}^{P}, \tilde{h} \circ \tilde{\tau}\right] .
$$

This map satisfies $\mathcal{P}(p)=\mathcal{P}(q)$ if and only if $p$ is equivalent to $q$ under the action of DB. Furthermore $\mathcal{P}$ is a holomorphic map which is locally a biholomorphism in the sense that for any point in $w \in T\left(\Sigma^{B}\right)$ there's an open set $U$ containing $w$ on which $\mathcal{P}$ is a biholomorphism onto an open subset of $\widetilde{T}^{P}\left(\Sigma^{P}\right)$.

Remark 2.9. The biholomorphism between $T^{B}\left(\Sigma^{B}\right) / \mathrm{DB}$ and $\widetilde{T}^{P}\left(\Sigma^{P}\right)$ is given explicitly by

$$
\begin{aligned}
G: T^{B}\left(\Sigma^{B}\right) / \mathrm{DB} & \rightarrow \widetilde{T}^{P}\left(\Sigma^{P}\right) \\
{\left[\Sigma^{B}, h, \Sigma_{1}^{B}\right] } & \mapsto \mathcal{P}\left(\left[\Sigma^{B}, h, \Sigma_{1}^{B}\right]\right) .
\end{aligned}
$$

That $G$ is well defined and a biholomorphism was established in [13. It is not possible to write the inverse explicitly, although we can say the following:

$$
G^{-1}\left(\left[\Sigma^{P}, f, \Sigma_{*}^{P}, \phi\right]\right)=\left[\Sigma^{B}, f_{\phi}, \Sigma_{*}^{P} \backslash \phi\left(\mathbb{D}_{0}^{n}\right)\right]
$$

where it is understood that the right hand side is a representative of a point in $T^{B}\left(\Sigma^{B}\right) / \mathrm{DB}$, and $f_{\phi}$ is a quasiconformal map satisfying (1) $f_{\phi}$ is homotopic to $f$, and (2) $h \circ \tau=\phi$ on $\partial \mathbb{D}$. Details are found in [13, Theorems 5.5 and 5.6]. 
Remark 2.10. Similarly, although $\mathcal{P}$ has a local holomorphic inverse, one cannot be completely explicit about it. Many of the difficulties in this paper can be traced to this fact. However, we are able to write the restriction of the inverse to specified holomorphic curves.

The local invertibility of $\mathcal{P}$ was established with the help of an existence theorem for quasiconformal maps, which is ultimately based on the $\lambda$-lemma; see 13 , Sections 4 and 5.4].

Next we define two fiber projections. Firstly, let

$$
\begin{aligned}
\mathcal{F}: \widetilde{T}^{P}\left(\Sigma^{P}\right) & \rightarrow T\left(\Sigma^{P}\right) \\
{\left[\Sigma^{P}, f, \Sigma_{1}^{P}, \phi\right] } & \mapsto\left[\Sigma^{P}, f, \Sigma_{1}^{P}\right] .
\end{aligned}
$$

Note that in $\left[13\right.$ we call this map $\mathcal{F}_{T}$. Secondly, we have the sewing map

$$
\begin{aligned}
\mathcal{C}: T\left(\Sigma^{B}\right) & \longrightarrow T\left(\Sigma^{P}\right) \\
{\left[\Sigma^{B}, h, \Sigma_{1}^{B}\right] } & \longmapsto\left[\Sigma^{B} \#_{\tau} \overline{\mathbb{D}}_{0}^{n}, \tilde{h}, \Sigma_{1}^{B} \#_{h \circ \tau} \overline{\mathbb{D}}_{0}^{n}\right] .
\end{aligned}
$$

It was proved in [13, Section 6] that $\mathcal{F} \circ \mathcal{P}=\mathcal{C}$ and $\mathcal{C}$ is a holomorphic map. Since $\mathcal{P}$ is a local biholomorphism it follows immediately that $\mathcal{F}$ is a holomorphic map.

We define the fibers by

$$
F_{B}\left(\left[\Sigma^{P}, f, \Sigma_{1}^{P}\right]\right)=\mathcal{C}^{-1}\left(\left[\Sigma^{P}, f, \Sigma_{1}^{P}\right]\right)
$$

and

$$
F_{P}\left(\left[\Sigma^{P}, f, \Sigma_{1}^{P}\right]\right)=\mathcal{F}^{-1}\left(\left[\Sigma^{P}, f, \Sigma_{1}^{P}\right]\right) .
$$

We will often denote a particular fiber by $F_{B}$ or $F_{P}$, if there is no fear of confusion. The action of DB preserves each fiber $F_{B}$ since $\mathcal{F} \circ \mathcal{P}=\mathcal{C}$ and thus we can conclude that $F_{P}=G\left(F_{B} / \mathrm{DB}\right)$.

Remark 2.11. $\mathcal{C}$ and $\mathcal{F}$, and hence the fibrations themselves, depend on the choice of $\tau$.

For the convenience of the reader we summarize this section with a theorem.

\section{Theorem 2.12.}

(1) $\widetilde{T}^{P}\left(\Sigma^{P}\right)$ possesses a complex structure.

(2) $\mathcal{P}$ is holomorphic, and for every point $w \in T\left(\Sigma^{B}\right)$, there is an open neighborhood $U$ of $w$ such that $\mathcal{P}$ is a biholomorphism onto its open image.

(3) $\mathcal{F} \circ \mathcal{P}=\mathcal{C}$.

(4) $\mathcal{F}$ and $\mathcal{C}$ are holomorphic and onto.

2.3. Fibers are complex submanifolds of $T\left(\Sigma^{B}\right)$. In this section we demonstrate that the fibers are complex submanifolds of $T\left(\Sigma^{B}\right)$. This requires the construction of local sections. The construction relies on a method of Gardiner 3] (see also Nag [11]), who used Schiffer variation to construct local coordinates on Teichmüller spaces of punctured Riemann surfaces of finite type.

We outline some necessary facts about complex submanifolds of Banach spaces. These can be found in for example [11, Section 1.6.2], and also [6] in the differentiable setting.

Let $E_{1}$ and $E_{2}$ be Banach spaces, and let $Y$ be a complex Banach manifold. Let $U_{1}$ and $U_{2}$ be open subsets of $E_{1}$ and $E_{2}$, respectively. We say that $g: U_{1} \times U_{2} \rightarrow Y$ is a projection, if there is a map $h: U_{1} \rightarrow Y$ such that $g=h \circ \operatorname{pr}_{1}$ where $\operatorname{pr}_{1}$ : $U_{1} \times U_{2} \rightarrow U_{1}$ is the projection onto the first component. 
Definition 2.13. Let $X$ and $Y$ be complex Banach manifolds. $f: X \rightarrow Y$ is a holomorphic submersion if $f$ is holomorphic and for all $x \in X$ there is a chart $(U, \phi)$ on $X$ with $x \in U$ and open sets $U_{1} \subset E_{1}$ and $U_{2} \subset E_{2}$ of Banach spaces $E_{1}$ and $E_{2}$, such that (1) $\phi: U \rightarrow U_{1} \times U_{2}$ is a biholomorphism and (2) there is a chart $(V, \psi)$ with $f(x) \in V$, such that $\psi \circ f \circ \phi^{-1}$ is a projection.

A holomorphic fiber space is defined as follows.

Definition 2.14. A holomorphic fiber space is a pair of complex Banach manifolds $(X, Y)$ together with a holomorphic, submersive and surjective map $\pi: X \rightarrow Y$.

It follows immediately from the definition of a submersion that the fibers of a submersion are complex submanifolds.

Lemma 2.15. A holomorphic submersion $F: X \rightarrow Y$ is an open mapping and the fibers $F^{-1}(y)$ are complex submanifolds of $X$.

We will use the following characterization of submersions.

Lemma 2.16. A holomorphic mapping $F: X \rightarrow Y$ between Banach spaces is submersive if and only if it possesses local holomorphic sections passing through every point $x \in X$.

We now briefly describe Schiffer variation of Riemann surfaces. For details see [3] or [11, Section 4.3]. Let $\Sigma$ be either a punctured Riemann surface of type $(g, n)$ or a bordered Riemann surface of type $(g, n)$. Let $(V, \zeta)$ be a holomorphic chart on $\Sigma$ such that $\overline{\mathbb{D}} \subset \zeta(V)$, and let $U=\zeta^{-1}(\mathbb{D})$ be a parametric disk on $\Sigma$.

For $\epsilon$ in a sufficiently small disk centered at $0 \in \mathbb{C}$, the map $v^{\epsilon}: \partial \mathbb{D} \rightarrow \mathbb{C}$ given by $v^{\epsilon}(z)=z+\epsilon / z$ is a biholomorphism on some neighborhood of $\partial \mathbb{D}$. Let $D^{\epsilon}$ denote the region bounded by $v^{\epsilon}(\partial \mathbb{D})$. We obtain a new Riemann surface $\Sigma^{\epsilon}$ as follows:

$$
\Sigma^{\epsilon}=(\Sigma \backslash U) \sqcup D^{\epsilon} / \sim
$$

where $x \in \partial U$ and $x^{\prime} \in \partial D^{\epsilon}$ are equivalent, $x \sim x^{\prime}$, if $x^{\prime}=v^{\epsilon} \circ \zeta(x)$. The complex structure on $\Sigma^{\epsilon}$ is compatible with $D^{\epsilon}$ and $\Sigma \backslash \bar{U}$.

The quasiconformal map $w^{\epsilon}: \overline{\mathbb{D}} \rightarrow D^{\epsilon}$ given by $w^{\epsilon}(z)=z+\epsilon \bar{z}$ has complex dilatation $\epsilon$. Let $\nu^{\epsilon}: \Sigma \rightarrow \Sigma^{\epsilon}$ be defined by

$$
\nu^{\epsilon}(x)= \begin{cases}x, & \text { if } x \in \Sigma \backslash U, \\ w^{\epsilon} \circ \zeta(x), & \text { if } x \in U .\end{cases}
$$

This map is quasiconformal with dilatation 0 on $\Sigma \backslash \bar{U}$ and dilatation $\epsilon d \bar{\zeta} / d \zeta$ on $U$.

Let $\Omega$ be some polydisc centered at $0 \in \mathbb{C}^{n}$ and $\epsilon=\left(\epsilon_{1}, \ldots, \epsilon_{n}\right) \in \Omega$. The above variation procedure can be applied to $n$ non-overlapping parametric disks $U_{1}, \ldots, U_{n}$ to obtain a Riemann surface $\Sigma^{\epsilon}$ and a quasiconformal map $\nu^{\epsilon}: \Sigma \rightarrow \Sigma^{\epsilon}$. We thus have a map

$$
\begin{aligned}
S: \Omega & \rightarrow T(\Sigma) \\
\epsilon & \mapsto\left[\Sigma, \nu^{\epsilon}, \Sigma^{\epsilon}\right] .
\end{aligned}
$$


The key result of [3] and [11, Theorem 4.3.2] is the following:

\section{Theorem 2.17.}

(1) The map $S$ is holomorphic for any type of Riemann surface $\Sigma$.

(2) Let $\Sigma^{P}$ be a punctured surface of type $(g, n)$, and let $d=3 g-3+n$. For an essentially arbitrary choice of parametric disks $U_{1}, \ldots, U_{n}$, the parameters $\left(\epsilon_{1}, \ldots, \epsilon_{d}\right)$ provide local holomorphic coordinates for $T\left(\Sigma^{P}\right)$ in a neighborhood of $[\Sigma, \mathrm{id}, \Sigma]$. That is, $S$ is a biholomorphism onto its image.

The first result follows directly from the fact that dilatation of $\nu^{\epsilon}$ is holomorphic in $\epsilon$. For the second result, it is non-trivial to prove that the variations give independent directions in $T\left(\Sigma^{P}\right)$.

Getting coordinates at an arbitrary point $\left[\Sigma^{P}, f, \Sigma_{1}^{P}\right]$ in Teichmüller space follows by applying a change of base surface biholomorphism from $T\left(\Sigma^{P}\right)$ to $T\left(\Sigma_{1}^{P}\right)$ in the following way. First we apply Schiffer variation to $\Sigma_{1}^{P}$. This gives a neighborhood of the base point in $T\left(\Sigma_{1}^{P}\right)$. Let $f_{*}: T\left(\Sigma_{1}^{P}\right) \rightarrow T\left(\Sigma^{P}\right)$ be the change of base surface biholomorphism corresponding to $f$. That is, $f_{*}\left(\left[\Sigma_{1}^{P}, \nu^{\epsilon}, \Sigma_{1}^{P}\right]\right)=\left[\Sigma^{P}, \nu^{\epsilon} \circ f, \Sigma_{1}^{P}\right]$. Thus, the image under $f_{*}$ of the Schiffer neighborhood in $T\left(\Sigma_{1}^{P}\right)$ is the Schiffer neighborhood of $\left[\Sigma^{P}, f, \Sigma_{1}^{P}\right] \in T\left(\Sigma^{P}\right)$.

We can now show that:

Theorem 2.18. The map $\mathcal{C}$ (2.4) possesses local holomorphic sections through every point.

Proof. Let $\left[\Sigma^{B}, f, \Sigma_{1}^{B}\right]$ be an arbitrary point in $T\left(\Sigma^{B}\right)$ and let $\left[\Sigma^{P}, \tilde{f}, \Sigma_{1}^{P}\right]=$ $\mathcal{C}\left[\Sigma^{B}, f, \Sigma_{1}^{B}\right]$ where $\mathcal{C}$ is the sewing map defined in (2.4). Recall that $\Sigma_{1}^{P}=$ $\Sigma_{1}^{B} \#_{f \circ \tau} \overline{\mathbb{D}}_{0}^{n}$. Let $d=3 g+3-n$. By Theorem 2.17 we can choose $d$ disjoint disks on $\Sigma_{1}^{B}$ such that performing Schiffer variation on $\Sigma_{1}^{P}$ using these disks results in a biholomorphic map

$$
\begin{aligned}
S: \Omega & \longrightarrow S(\Omega) \subset T\left(\Sigma^{P}\right) \\
\epsilon & \longmapsto\left[\Sigma^{P},(\tilde{f})^{\epsilon}, \Sigma_{1}^{P, \epsilon}\right],
\end{aligned}
$$

where $(\tilde{f})^{\epsilon}=\nu^{\epsilon} \circ \tilde{f}, \epsilon=\left(\epsilon_{1}, \ldots, \epsilon_{d}\right)$, and $\Omega$ is an open neighborhood of $0 \in \mathbb{C}^{d}$.

Performing the Schiffer variation on $\Sigma_{1}^{B}$ using the same disks produces a holomorphic map

$$
\begin{aligned}
S^{B}: \Omega & \longrightarrow T\left(\Sigma^{B}\right) \\
\epsilon & \longmapsto\left[\Sigma^{B}, f^{\epsilon}, \Sigma_{1}^{B, \epsilon}\right],
\end{aligned}
$$

where $f^{\epsilon}=\nu^{\epsilon} \circ f$.

By Theorem 2.17, $\eta=S^{B} \circ S^{-1}: S(\Omega) \rightarrow T\left(\Sigma^{B}\right)$ is holomorphic. To show that it is a section of $\mathcal{C}$ through $\left[\Sigma^{B}, f, \Sigma_{1}^{B}\right]$, it remains to show that $\mathcal{C} \circ \eta$ is the identity.

Note that $f=f^{\epsilon}$ on $\partial \Sigma$, and by definition of $\tilde{f},(\tilde{f})^{\epsilon}=\tilde{f} \epsilon$. Because the disks on which to perform Schiffer variation were chosen to be away from the caps of $\Sigma_{1}^{P}$,

$$
\Sigma_{1}^{B, \epsilon} \#_{f^{\epsilon} \circ \tau} \overline{\mathbb{D}}_{0}^{n}=\Sigma_{1}^{P, \epsilon} .
$$

So, we have

$$
\begin{aligned}
(\mathcal{C} \circ \eta)\left(\left[\Sigma^{P},(\tilde{f})^{\epsilon}, \Sigma_{1}^{P, \epsilon}\right]\right) & =\mathcal{C}\left(\left[\Sigma^{B}, f^{\epsilon}, \Sigma_{1}^{B, \epsilon}\right]\right)=\left[\Sigma^{P}, \tilde{f} \epsilon, \Sigma_{1}^{B, \epsilon} \#_{f^{\epsilon} \circ \tau} \overline{\mathbb{D}}_{0}^{n}\right] \\
& =\left[\Sigma^{P},(\tilde{f})^{\epsilon}, \Sigma_{1}^{P, \epsilon}\right]
\end{aligned}
$$

and thus $\mathcal{C} \circ \eta$ is the identity. 
We now have the following key results.

Corollary 2.19. The Teichmüller space $T\left(\Sigma^{B}\right)$ is a holomorphic fiber space over $T\left(\Sigma^{P}\right)$ with fiber structure given by the sewing map

$$
\mathcal{C}: T\left(\Sigma^{B}\right) \longmapsto T\left(\Sigma^{P}\right) .
$$

Proof. Theorem 2.12 states that $\mathcal{C}$ is holomorphic and onto. Lemma 2.16 and Theorem 2.18 show that $\mathcal{C}$ is a submersion.

From Lemma 2.15 we obtain:

Corollary 2.20. The fibers $F_{B}$ are complex submanifolds of $T\left(\Sigma^{B}\right)$.

Corollary 2.21. The fibers $F_{P}$ are complex submanifolds of $\widetilde{T}^{P}\left(\Sigma^{P}\right)$.

Proof. From Theorem 2.12, $\mathcal{F}$ is holomorphic. It possesses local holomorphic sections, since if $\rho: T\left(\Sigma^{P}\right) \rightarrow T\left(\Sigma^{B}\right)$ is a local holomorphic section of $\mathcal{C}$, then $\mathcal{P} \circ \rho$ is a local holomorphic section of $\mathcal{F}$.

\section{LOCAL MODEL OF FIBERS}

3.1. Complex structure on $\mathcal{O}_{\mathrm{qc}}\left(\Sigma_{1}^{P}\right)$. The authors defined a complex structure on $\mathcal{O}_{\text {qc }}\left(\Sigma_{1}^{P}\right)$ in [14, with the help of a model of the universal Teichmüller curve due to Teo [17. We outline the definition of the complex structure in this section.

The complex structure $\mathcal{O}_{\mathrm{qc}}\left(\Sigma_{1}^{P}\right)$ is locally biholomorphic to $\mathcal{O}_{\mathrm{qc}}^{n}=\mathcal{O}_{\mathrm{qc}} \times \cdots \times \mathcal{O}_{\mathrm{qc}}$. So we must first describe the complex structure on $\mathcal{O}_{\text {qc }}$. To do this, we define an injection of $\mathcal{O}_{\mathrm{qc}}$ onto an open subset of a Banach space. Consider the Banach space

$$
A_{\infty}^{1}(\mathbb{D})=\left\{v(z): \mathbb{D} \rightarrow \mathbb{C} \mid v \text { holomorphic, }\|v\|_{1, \infty}=\sup _{z \in \mathbb{D}}\left(1-|z|^{2}\right)|v(z)|<\infty\right\} .
$$

For $f \in \mathcal{O}_{\mathrm{qc}}$ define

$$
\mathcal{A}(f)=\frac{f^{\prime \prime}(z)}{f^{\prime}(z)} .
$$

We then have a natural one-to-one map

$$
\begin{aligned}
\chi: \mathcal{O}_{\mathrm{qc}} & \rightarrow A_{\infty}^{1}(\mathbb{D}) \oplus \mathbb{C} \\
f & \mapsto\left(\mathcal{A}(f), f^{\prime}(0)\right),
\end{aligned}
$$

where $A_{\infty}^{1}(\mathbb{D}) \oplus \mathbb{C}$ is a Banach space with the direct sum norm

$$
\|(\phi, c)\|=\|\phi\|_{1, \infty}+|c| .
$$

It was shown in [14 that the image of $\mathcal{O}_{\mathrm{qc}}$ is open and thus $\mathcal{O}_{\mathrm{qc}}$ inherits a complex structure from $A_{\infty}^{1}(\mathbb{D}) \oplus \mathbb{C}$.

We now describe the complex structure on $\mathcal{O}_{\mathrm{qc}}\left(\Sigma_{1}^{P}\right)$ in terms of local charts into $\mathcal{O}_{\mathrm{qc}}^{n}$. Fix a point $\left(\phi_{1}, \ldots, \phi_{n}\right) \in \mathcal{O}_{\mathrm{qc}}\left(\Sigma_{1}^{P}\right)$. For $i=1, \ldots, n$, let $D_{i}=\phi_{i}(\mathbb{D})$ be open sets in $\Sigma_{1}^{P}$. Choose domains $B_{i} \subset \Sigma_{1}^{P}$ with the following properties: (1) $\phi_{i}(\overline{\mathbb{D}}) \subset B_{i},(2) B_{i} \cap B_{j}=\emptyset$ for $i \neq j$ and (3) $B_{i}$ are open and simply connected. Let $\zeta_{i}: B_{i} \rightarrow \mathbb{C}$ be a local biholomorphic parameter such that $\zeta_{i}\left(p_{i}\right)=0$. We then have that $\zeta_{i} \circ \phi_{i} \in \mathcal{O}_{\mathrm{qc}}$.

Let $K_{i}$ be a compact set, which is the closure of an open set containing $\zeta_{i}$ 。 $\phi_{i}(\overline{\mathbb{D}})$, such that $\zeta_{i}^{-1}\left(K_{i}\right) \subset B_{i}$. By [14, Corollary 3.5], for each $i$ there is an open neighborhood $U_{i}$ of $\zeta_{i} \circ \phi_{i}$ in $\mathcal{O}_{\mathrm{qc}}$ such that $\psi_{i}(\overline{\mathbb{D}}) \subset K_{i}$ for all $\psi_{i} \in U_{i}$, and so $\left(\zeta_{1}^{-1} \circ \psi_{1}, \ldots, \zeta_{n}^{-1} \circ \psi_{n}\right)$ is an element of $\mathcal{O}_{\mathrm{qc}}\left(\Sigma_{1}^{P}\right)$. 

Let

Thus, $U_{1} \times \cdots \times U_{n}$ is an open subset of $\mathcal{O}_{\mathrm{qc}} \times \cdots \times \mathcal{O}_{\mathrm{qc}}$ with the product topology.

$$
V_{i}=\left\{\zeta_{i}^{-1} \circ \psi_{i} \mid \psi_{i} \in U_{i}\right\}
$$

then sets of the form $V=V_{1} \times \cdots \times V_{n}$ form a base of the topology of $\mathcal{O}_{\mathrm{qc}}\left(\Sigma_{1}^{P}\right)$. Let

$$
\begin{aligned}
T_{i}: V_{i} & \rightarrow \mathcal{O}_{\mathrm{qc}} \\
g & \mapsto \zeta_{i} \circ g
\end{aligned}
$$

and note that $T_{i}\left(V_{i}\right)=U_{i}$. The local coordinates

$$
T=\left(T_{1}, \ldots, T_{n}\right): V \rightarrow U
$$

define a complex Banach manifold structure on $\mathcal{O}_{\mathrm{qc}}\left(\Sigma_{1}^{P}\right)$.

Remark 3.1. In fact, fixing $B_{i}$ and $\zeta_{i}, i=1, \ldots, n, T$ is a valid chart on the set of all elements of $\mathcal{O}_{\mathrm{qc}}\left(\Sigma_{1}^{P}\right)$ which map all $n$ copies of the disk into the corresponding set $B_{i}$. To see this, let $U_{i}=\left\{\psi_{i} \in \mathcal{O}_{\mathrm{qc}} \mid \psi_{i}(\overline{\mathbb{D}}) \subset \zeta_{i}\left(B_{i}\right)\right\}$ and observe that $U=$ $U_{1} \times \cdots \times U_{n}$ is open by [14, Corollary 3.5]. Furthermore, the set $V=\{\phi \in$ $\left.\mathcal{O}_{\mathrm{qc}}\left(\Sigma_{1}^{P}\right) \mid \phi_{i}(\overline{\mathbb{D}}) \subset B_{i}\right\}$ is open since every point is contained in an open set of the form described above. The map $T$ is clearly a biholomorphism on all of $V$. Hence, $(T, V)$ is a local coordinate.

3.2. The biholomorphism $L: \mathcal{O}_{\mathrm{qc}}\left(\Sigma_{*}^{P}\right) \rightarrow F_{P}\left(\left[\Sigma^{P}, f, \Sigma_{*}^{P}\right]\right)$. Fix a point $\left[\Sigma^{P}, f, \Sigma_{*}^{P}\right]$ $\in T\left(\Sigma^{P}\right)$ and recall from Section 2.2 that $F_{P}\left(\left[\Sigma^{P}, f, \Sigma_{*}^{P}\right]\right) \subset \widetilde{T}^{P}\left(\Sigma^{P}\right)$ is the fiber over this point. Define the map

$$
\begin{aligned}
L: \mathcal{O}_{\mathrm{qc}}\left(\Sigma_{*}^{P}\right) & \rightarrow F_{P}\left(\left[\Sigma^{P}, f, \Sigma_{*}^{P}\right]\right) \\
\phi & \mapsto\left[\Sigma^{P}, f, \Sigma_{*}^{P}, \phi\right] .
\end{aligned}
$$

Our goal is to prove the following theorem.

Theorem 3.2. The map $L: \mathcal{O}_{\mathrm{qc}}\left(\Sigma_{*}^{P}\right) \rightarrow F_{P}\left(\left[\Sigma^{P}, f, \Sigma_{*}^{P}\right]\right)$ is a biholomorphism.

Proof. We will prove this in three stages: first, we show that $L$ is a bijection onto $F_{P}$ (see Lemma 3.9); second, that $L$ is holomorphic (see Lemma 3.11). Lastly, we show that the inverse is holomorphic.

To show that the inverse is holomorphic, in Section 3.4 we will construct near any point in $T\left(\Sigma^{B}\right)$ a local inverse $\Lambda$ to the lift $\mathcal{P}^{-1} \circ L$ where $\mathcal{P}^{-1}$ is a local inverse of $\mathcal{P}$. By Theorem 2.12 it is enough to show that $\Lambda$ is holomorphic, which we will establish in Theorem 3.23

Theorem 3.2 has the following consequences.

Corollary 3.3. Fix any $\left[\Sigma^{P}, f, \Sigma_{*}^{P}\right] \in T\left(\Sigma^{P}\right)$. The map

$$
\begin{aligned}
\mathcal{O}_{\mathrm{qc}}\left(\Sigma_{*}^{P}\right) & \rightarrow F_{B}\left(\left[\Sigma^{P}, f, \Sigma_{*}^{P}\right]\right) / \mathrm{DB} \\
\phi & \mapsto\left[\Sigma^{B}, f_{\phi}, \Sigma_{*}^{P} \backslash \phi\left(\mathbb{D}_{0}^{n}\right)\right]
\end{aligned}
$$

is a biholomorphism, where $f_{\phi}$ is the restriction of a map $f_{\phi}^{\prime}: \Sigma^{P} \rightarrow \Sigma_{1}^{P}$ which is homotopic to $f$ and satisfies $f_{\phi}^{\prime} \circ \tilde{\tau}=\phi$.

Proof. This follows from Theorem 3.2 and Remark 2.9 .

Corollary 3.4. The fibers $F_{B}\left(\left[\Sigma^{P}, f, \Sigma_{1}^{P}\right]\right)$ and $F_{P}\left(\left[\Sigma^{P}, f, \Sigma_{1}^{P}\right]\right)$ are locally biholomorphic to $\mathcal{O}_{\mathrm{qc}}^{n}$ for any $\left[\Sigma^{P}, f, \Sigma_{1}^{P}\right]$ via the maps $T \circ L^{-1} \circ \mathcal{P}$ and $T \circ L^{-1}$, respectively. 
In the next few sections we require some facts regarding infinite-dimensional holomorphy (see for example [2], [8, V.5.1] or [11, Section 1.6]). Let $E$ and $F$ be Banach spaces and let $U$ be an open subset of $E$.

Definition 3.5. A map $f: U \rightarrow F$ is holomorphic if for each $x_{0} \in U$ there is a continuous complex linear map $D f\left(x_{0}\right): E \rightarrow F$ such that

$$
\lim _{h \rightarrow 0} \frac{\left\|f\left(x_{0}+h\right)-f\left(x_{0}\right)-D f\left(x_{0}\right)(h)\right\|_{F}}{\|h\|_{E}}=0 .
$$

Definition 3.6. A map $f: U \rightarrow F$ is called Gâteaux holomorphic if $f$ is holomorphic on complex lines. That is, if for all $a \in U$ and all $x \in E$, the map $z \mapsto f(a+z x)$ is holomorphic on $\{z \in \mathbb{C} \mid a+z x \in U\}$.

Theorem 3.7 (see [2, p. 198]). Let $f: U \rightarrow F$. The following are equivalent.

(1) $f$ is holomorphic.

(2) $f$ is Gâteaux-holomorphic and continuous.

(3) $f$ is Gâteaux-holomorphic and locally bounded on $U$.

A subset $H$ of the (continuous) dual space $F^{\prime}$ is called separating if for all nonzero $x \in F$ there exists $\alpha \in H$ such that $\alpha(x) \neq 0$. The following theorem gives another characterization of holomorphicity; see [4] for a statement in a more general setting.

Theorem 3.8. Let $f: \Omega \rightarrow F$ be a function on a domain $\Omega$ in $\mathbb{C}$. If

(1) $\alpha \circ f$ is holomorphic for each continuous linear functional $\alpha$ from a separating subset of the dual space $F^{\prime}$, and

(2) $f$ is locally bounded,

then $f$ is holomorphic.

We now turn to first step in the proof of Theorem $\underline{3.2}$

Lemma 3.9. $L$ is a bijection between $\mathcal{O}_{\mathrm{qc}}\left(\Sigma_{*}^{P}\right)$ and $F_{P}\left(\left[\Sigma^{P}, f, \Sigma_{*}^{P}\right]\right)$.

Proof. By the definitions of $\widetilde{T}^{P}\left(\Sigma^{P}\right)$ and $T\left(\Sigma^{P}\right)$, every element in $F_{P}$ has a representative of the form $\left[\Sigma^{P}, \tilde{f}, \Sigma_{*}^{P}, \phi\right]$. $L$ is thus clearly a surjection.

Assume that $L(\phi)=L(\psi)$. If $\mathcal{F}\left(\left[\Sigma^{P}, f, \Sigma_{*}^{P}, \phi\right]\right)=\mathcal{F}\left(\left[\Sigma^{P}, f, \Sigma_{*}^{P}, \psi\right]\right)$, then there exists a biholomorphism $\sigma: \Sigma_{*}^{P} \rightarrow \Sigma_{*}^{P}$ that is homotopic to the identity. Since $2 g-2+n>0$ there are no non-trivial automorphisms which are homotopic to the identity. Thus $\sigma$ is the identity and so $\phi=\psi$.

Remark 3.10. The bijection is not canonical since $\mathcal{O}_{\mathrm{qc}}\left(\Sigma_{*}^{P}\right)$ depends on the choice of representative in the Teichmüller equivalence class. However, if $\left[\Sigma^{P}, f_{1}, \Sigma_{1}^{P}\right]=$ $\left[\Sigma^{P}, f_{2}, \Sigma_{2}^{P}\right]$ and $\sigma: \Sigma_{1}^{P} \rightarrow \Sigma_{2}^{P}$ is the biholomorphism realizing the equivalence, then we have the bijection $\sigma^{*}: \mathcal{O}_{\mathrm{qc}}\left(\Sigma_{1}^{P}\right) \rightarrow \mathcal{O}_{\mathrm{qc}}\left(\Sigma_{2}^{P}\right)$ defined by $\sigma^{*}\left(\phi_{1}\right)=\sigma \circ \phi_{1}$, and $L_{1}=L_{2} \circ \sigma^{*}$. It will follow from Theorem 3.2 (once the proof is complete) that $\mathcal{O}_{\mathrm{qc}}\left(\Sigma_{1}^{P}\right)$ and $\mathcal{O}_{\mathrm{qc}}\left(\Sigma_{2}^{P}\right)$ are biholomorphic under $\sigma^{*}$.

\section{3. $L$ is holomorphic.}

Lemma 3.11. L is holomorphic.

Proof. Fix $\phi_{0} \in \mathcal{O}_{\mathrm{qc}}\left(\Sigma_{*}^{P}\right)$. We will show that $L$ is holomorphic in a neighborhood of $\phi_{0}$ by first proving that $L$ is Gâteaux holomorphic and then that the lift of $L$ to $L_{-1,1}^{\infty}\left(\Sigma^{B}\right)_{1}$ is locally bounded. 
To simplify notation we assume that $\Sigma^{P}$ has only one puncture, $p_{1}$. The proof of the general case is identical with the exception of the notation. Let $(T, V)$ be a chart on $\mathcal{O}_{\mathrm{qc}}\left(\Sigma_{*}^{P}\right)$ containing $\phi_{0}$ and let $B=B_{1}, D=D_{1}$ and $\zeta=\zeta_{1}$ be as in Section 3.1. Recall that the map $\chi$ (3.1) defines the complex structure on $\mathcal{O}_{\mathrm{qc}}$.

To show that $L$ is Gâteaux holomorphic we must show that

$$
L \circ T^{-1} \circ \chi^{-1}: A_{\infty}^{1}(\mathbb{D}) \oplus \mathbb{C} \rightarrow \widetilde{T}^{P}\left(\Sigma^{P}\right)
$$

is Gâteaux holomorphic. Since the complex structure on $\widetilde{T}^{P}\left(\Sigma^{P}\right)$ is obtained from $T\left(\Sigma^{B}\right)$ we proceed by producing a lift of $L \circ T^{-1} \circ \chi^{-1}$ to $T\left(\Sigma^{B}\right)$.

Let $\psi_{0}=T\left(\phi_{0}\right)=\zeta \circ \phi_{0} \in \mathcal{O}_{\mathrm{qc}}, u_{0}=\mathcal{A}\left(\psi_{0}\right) \in A_{1}^{\infty}$, and choose an element $(v, c) \in A_{1}^{\infty}(\mathbb{D}) \oplus \mathbb{C}$. Let $N$ be an open neighborhood of $0 \in \mathbb{C}$, and consider the map $N \rightarrow \chi(T(V)) \subset A_{1}^{\infty} \oplus \mathbb{C}$ given by $t \mapsto\left(u_{0}+t v, q_{t}\right)$ where $q_{t}=\psi_{0}^{\prime}(0)+t c$. That is, we have a complex line through $\chi\left(\psi_{0}\right)=\left(u_{0}, \psi_{0}^{\prime}(0)\right)$.

Let

$$
\psi_{t}=\chi^{-1}\left(u_{0}+t v, q_{t}\right)
$$

From the definition (3.1) of $\chi$ we have a differential equation for $\psi_{t}$, whose solution is

$$
\psi_{t}(z)=\frac{q_{t}}{\psi_{0}^{\prime}(0)} \int_{0}^{z} \psi_{0}^{\prime}(\xi) \exp \left(t \int_{0}^{\xi} g(w) d w\right) d \xi
$$

It is clear from this expression that $\psi_{t}(z)$ is holomorphic in $t$ for fixed $z \in \mathbb{D}$.

Now, $\psi_{t} \circ \psi_{0}^{-1}$ is a holomorphic motion of $\psi_{0}(\mathbb{D})$. By the extended lambda-lemma [19] it extends to a holomorphic motion of $\mathbb{C}$, and in particular the continuous extension of $\psi_{t} \circ \psi_{0}^{-1}$ to $\psi_{0}(\overline{\mathbb{D}})$ is a holomorphic motion. Thus $\psi_{t} \circ \psi_{0}^{-1}$ restricted to $\psi_{0}(\partial \mathbb{D})$ is a holomorphic motion.

Let $\phi_{t}=T^{-1}\left(\psi_{t}\right)=\zeta^{-1} \circ \psi_{t}$, and let $A_{t}=\bar{B} \backslash \phi_{t}(\mathbb{D})$ be the annular region on $\Sigma_{*}^{P}$ bounded by $\partial B$ and $\phi_{t}(\partial \mathbb{D})$. Applying [13, Lemma 7.1] we obtain a holomorphic motion $H_{t}: \zeta\left(A_{0}\right) \rightarrow \zeta\left(A_{t}\right)$ such that $\left.H_{t}\right|_{\zeta(\partial B)}$ is the identity and $H_{t_{\psi_{0}}(\partial \mathbb{D})}=$ $\psi_{t} \circ \psi_{0}^{-1}$. by

Let $\Sigma_{*, t}^{B}=\Sigma_{*}^{P} \backslash \phi_{t}(\mathbb{D})$. By [13, Proposition 7.1] the map $F_{t}: \Sigma_{*, 0}^{B} \rightarrow \Sigma_{*, t}^{B}$ defined

$$
F_{t}= \begin{cases}\mathrm{id}, & \text { on } \Sigma_{*}^{B} \backslash A_{0}, \\ \zeta^{-1} \circ H_{t} \circ \zeta, & \text { on } A_{0}\end{cases}
$$

is quasiconformal and holomorphic in $t$ for fixed $z$. On $\phi_{0}(\partial \mathbb{D}), F_{t}=\zeta^{-1} \circ \psi_{t} \circ$ $\psi_{0}^{-1} \circ \zeta=\phi_{t} \circ \phi_{0}^{-1}$. From [13, Proposition 7.2], $t \mapsto \mu\left(F_{t}\right)$ is a holomorphic function $N \rightarrow L_{-1,1}^{\infty}\left(\Sigma_{*, 0}^{B}\right)_{1}$. Therefore, by the holomorphicity of the fundamental projection, $t \mapsto\left[\Sigma_{*, 0}^{B}, F_{t}, \Sigma_{*, t}^{B}\right]$ is holomorphic.

Let $\left[\Sigma^{B}, h_{0}, \Sigma_{*, 0}^{B}\right]=\mathcal{P}^{-1}\left(\left[\Sigma^{P}, f, \Sigma_{*}^{P}, \phi_{0}\right]\right)$ where $\mathcal{P}^{-1}$ is a local inverse of $\mathcal{P}$ and $\tilde{h}_{0} \circ \tilde{\tau}=\phi_{0}$ from the definition of $\mathcal{P}$ in (2.2). For $g: \Sigma^{B} \rightarrow \Sigma_{*, 0}^{B}$, let

$$
\begin{aligned}
g_{*}: T\left(\Sigma_{*, 0}^{B}\right) & \rightarrow T\left(\Sigma^{B}\right) \\
{\left[\Sigma_{*, 0}^{B}, h_{1}, \Sigma_{1}^{B}\right] } & \mapsto\left[\Sigma^{B}, h_{1} \circ g, \Sigma_{1}^{B}\right]
\end{aligned}
$$

be the change of base point biholomorphism (see [11, Sections 2.3.1 and 3.2.5]). In particular, $g_{*}\left(\left[\Sigma_{*, 0}^{B}, F_{t}, \Sigma_{*, t}^{B}\right]\right)=\left[\Sigma^{B}, F_{t} \circ g, \Sigma_{*, t}^{B}\right]$ is a biholomorphism, and therefore the map $t \mapsto\left[\Sigma^{B}, F_{t} \circ h_{0}, \Sigma_{*, t}^{B}\right]$ from $N$ into $T\left(\Sigma^{B}\right)$ is holomorphic. Moreover, $t \mapsto \mu\left(F_{t} \circ h_{0}\right)$ is also holomorphic. 
From the boundary values of $F_{t}$ and $h_{0}$ we see that $F_{t} \circ h_{0} \circ \tau=\phi_{t}$ on $\partial \mathbb{D}$. Furthermore, extending $F_{t}, h_{0}$ and $\tau$ by the identity to the caps as in equation (2.1), we have that $\tilde{F}_{t} \circ \tilde{h}_{0} \circ \tilde{\tau}$ is homotopic to $f$ (after identifying $\Sigma_{*, t}^{B} \# \overline{\mathbb{D}}_{0}$ with $\left.\Sigma_{*}^{P}\right)$. Thus

$$
\mathcal{P}\left(\left[\Sigma^{B}, F_{t} \circ h_{0}, \Sigma_{*, t}^{B}\right]\right)=\left[\Sigma^{P}, \tilde{F}_{t} \circ \tilde{h}_{0}, \Sigma_{*, t}^{B} \# \phi_{t} \overline{\mathbb{D}}_{0}, \phi_{t}\right]=\left[\Sigma^{P}, f, \Sigma_{*}^{P}, \phi_{t}\right]
$$

and so

$$
\begin{aligned}
N & \rightarrow \widetilde{T}\left(\Sigma^{P}\right) \\
t & \mapsto\left(L \circ T^{-1} \circ \chi^{-1}\right)\left(h_{0}+t g, q_{t}\right)=\left[\Sigma^{P}, f, \Sigma_{*}^{P}, \phi_{t}\right]
\end{aligned}
$$

is holomorphic. That is, $L \circ T^{-1} \circ \chi^{-1}$ is Gâteaux holomorphic.

We have that (1) the fundamental projection $\Phi: L_{-1,1}^{\infty}\left(\Sigma^{B}\right)_{1} \rightarrow T\left(\Sigma^{B}\right)$ is holomorphic and possesses local holomorphic sections and $(2) \mathcal{P}: T\left(\Sigma^{B}\right) \rightarrow \widetilde{T}^{P}\left(\Sigma^{P}\right)$ is holomorphic and possesses local holomorphic sections. Thus there is an open neighborhood $M$ of $\left[\Sigma^{P}, f, \Sigma_{*}^{P}, \phi_{0}\right] \in \widetilde{T}^{P}\left(\Sigma^{P}\right)$ and a local holomorphic section $\sigma: M \rightarrow L_{-1,1}^{\infty}\left(\Sigma^{B}\right)$ of $\mathcal{P} \circ \Phi$. Continuity in $t$ guarantees that for $|t|$ sufficiently small, $\left[\Sigma^{P}, f, \Sigma_{*}^{P}, \phi_{t}\right] \subset M$. The function $\sigma \circ L$ is Gâteaux holomorphic and locally bounded since $\sigma$ maps into the open unit ball. Thus, by Theorem [3.7, $\sigma \circ L$ is holomorphic, and therefore $L=\mathcal{P} \circ \Phi \circ \sigma \circ L$ is holomorphic.

Remark 3.12. For any fixed $z \in \mathbb{D}$, the point evaluation map $\mathcal{O}_{\mathrm{qc}} \rightarrow \mathbb{C}$ given by $f \mapsto f(z)$ is holomorphic. The proof is an immediate consequence of Theorem 3.7 noting that Gâteaux holomorphy follows from equation (3.2), and continuity is proved in [14, Corollary 3.4]. This result is also included in the proof of [14, Lemma 3.10] but it is not mentioned explicitly.

3.4. The local inverse of $L$. In order to show that $L^{-1}$ is biholomorphic, we show that $\mathcal{P}^{-1} \circ L: \mathcal{O}_{\mathrm{qc}}\left(\Sigma_{*}^{P}\right) \rightarrow T\left(\Sigma^{B}\right)$ has a local holomorphic inverse for any local inverse $\mathcal{P}^{-1}$ of $\mathcal{P}$. The description of the inverse to $\mathcal{P}^{-1} \circ L$ is somewhat lengthy and deserves its own section. In fact we are only able to explicitly describe the inverse on specified holomorphic curves. The source of the trouble can be partly traced to the fact that local inverses of $\mathcal{P}$ cannot be explicitly defined (see Remark 2.10).

It is necessary to make the following change of base point. Recall that $\Sigma^{P}=$ $\Sigma^{B} \#_{\tau} \overline{\mathbb{D}}_{0}^{n}$ as in Section 2.2 , which we now think of as a punctured surface. Let $\mathbb{U}$ be the upper half-plane, and choose a Fuchsian group $G$ such that $\Sigma_{G}=\mathbb{U} / G$ is an $n$-punctured surface biholomorphic to $\Sigma^{P}$. Let

$$
\alpha: \Sigma^{P} \rightarrow \Sigma_{G}
$$

be a fixed biholomorphism.

Let $A$ be an open set of $T\left(\Sigma^{B}\right)$ such that $\left.\mathcal{P}\right|_{A}$ is a biholomorphism. Let

$$
A_{B}=F_{B} \cap A,
$$

where $F_{B}$ is the fiber in $T\left(\Sigma^{B}\right)$ above the fixed point $\left[\Sigma^{P}, f, \Sigma_{*}^{P}\right]$ (see Section 2.2 ).

Given $\left[\Sigma^{B}, h, \Sigma_{1}^{B}\right] \in A_{B}$ we have that $\mathcal{P}\left(\left[\Sigma^{B}, h, \Sigma_{1}^{B}\right]\right)=\left(\left[\Sigma^{P}, \tilde{h}, \Sigma_{1}^{B} \#_{h \circ \tau} \overline{\mathbb{D}}_{0}^{n}\right]\right)$. The change of base point biholomorphism $\left(\alpha^{*}\right)^{-1}: T\left(\Sigma^{P}\right) \rightarrow T\left(\Sigma_{G}\right)$ is defined by

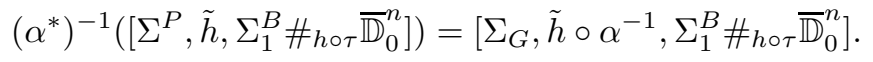


Now define the Beltrami differential

$$
\mu=\mu\left(\tilde{h} \circ \alpha^{-1}\right)= \begin{cases}\mu\left(h \circ \alpha^{-1}\right), & \text { on } \alpha\left(\Sigma^{B}\right) \subset \Sigma_{G}, \\ 0, & \text { on } \alpha(\mathbb{D}),\end{cases}
$$

in $L_{-1,1}^{\infty}\left(\Sigma_{G}\right)_{1}$. Let $L^{\infty}(\mathbb{U}, G)_{1}$ be the space of Beltrami differentials compatible with $G$, and following [11, p. 51] we identify $\mu$ with its unique lift to $L^{\infty}(\mathbb{U}, G)_{1}$. Let $w^{\mu}$ be the unique solution to the Beltrami equation on $\mathbb{C}$ fixing 0,1 and $\infty$ and having dilatation 0 on the lower half-plane. Let $G^{\mu}=w^{\mu} \circ G \circ\left(w^{\mu}\right)^{-1}$ and

$$
\Sigma^{\mu}=w^{\mu}(\mathbb{U}) / G^{\mu} .
$$

Let $T(G)=L^{\infty}(\mathbb{U}, G)_{1} / \sim$, where $\mu \sim \nu$ if and only if $w^{\mu}=w^{\nu}$ on $\mathbb{R}$, be the Teichmüller space of $G$. Let $\pi^{\mu}: w^{\mu}(\mathbb{U}) \rightarrow w^{\mu}(\mathbb{U}) / G^{\mu}$ be the canonical projection. It is a standard fact (see [11, Sections 2.2.2 and 3.3.1]) that:

Lemma 3.13. If $[\mu]=[\nu]$ in $T(G)$, then $w^{\mu}(\mathbb{U})=w^{\nu}(\mathbb{U}), G^{\mu}=G^{\nu}$, and $\pi^{\mu}=\pi^{\nu}$.

Proposition 3.14. The equivalence class $[\mu]$ and the Riemann surface $\Sigma^{\mu}$ are independent of $\left[\Sigma^{B}, h, \Sigma_{1}^{B}\right] \in A_{B}$.

Proof. By definition of the fiber $F_{B},\left[\Sigma^{P}, f, \Sigma_{*}^{P}\right]=\left[\Sigma^{P}, \tilde{h}, \Sigma_{1}^{B} \#_{h \circ \tau} \overline{\mathbb{D}}_{0}\right]$, and applying $\left(\alpha^{*}\right)^{-1}$ leads to

$$
\left[\Sigma_{G}, f \circ \alpha^{-1}, \Sigma_{*}^{P}\right]=\left[\Sigma_{G}, \tilde{h} \circ \alpha^{-1}, \Sigma_{1}^{B} \#{ }_{h \circ \tau} \overline{\mathbb{D}}_{0}\right] .
$$

Therefore $\mu=\mu\left(\tilde{h} \circ \alpha^{-1}\right)$ is equivalent to the fixed element $\mu\left(f \circ \alpha^{-1}\right)$. The result now follows from Lemma 3.13 .

We may therefore let $\sigma: \Sigma^{\mu} \rightarrow \Sigma_{*}^{P}$ be a fixed biholomorphism, and define

$$
\begin{aligned}
\Lambda: A_{B} & \rightarrow \mathcal{O}_{\mathrm{qc}}\left(\Sigma_{*}^{P}\right) \\
{\left[\Sigma^{B}, h, \Sigma_{1}^{B}\right] } & \mapsto \sigma \circ f^{\mu} \circ \alpha \circ \tilde{\tau}
\end{aligned}
$$

where $\mu=\mu\left(\tilde{h} \circ \alpha^{-1}\right)$ and $f^{\mu}$ is the unique quotient map

$$
f^{\mu}: \Sigma_{G} \rightarrow \Sigma^{\mu}
$$

corresponding to $w^{\mu}$.

Remark 3.15. The argument above also shows that for any $\left[\Sigma^{B}, h_{1}, \Sigma_{1}^{B}\right]$ and $\left[\Sigma^{B}, h_{2}, \Sigma_{2}^{B}\right]$ in $A_{B}$, the corresponding $w^{\mu_{1}}$ and $w^{\mu_{2}}$ are homotopic rel $\partial \mathbb{U}$, and similarly $f^{\mu_{1}}$ and $f^{\mu_{2}}$ are homotopic in $\Sigma_{G}$ [11, Sections 2.2.2 and 3.3.1].

Remark 3.16. The map

$$
\begin{aligned}
L_{-1,1}^{\infty}\left(\Sigma^{B}\right) & \rightarrow L_{-1,1}^{\infty}\left(\Sigma_{G}\right) \\
\mu(h) & \mapsto \mu\left(\tilde{h} \circ \alpha^{-1}\right)
\end{aligned}
$$

is holomorphic. This follows from the fact that $\mu(h) \mapsto \mu(\tilde{h})$ is holomorphic (see [13, Lemma 6.2]), and the map $\mu(\tilde{h}) \mapsto \mu\left(\tilde{h} \circ \alpha^{-1}\right)$ is holomorphic by holomorphicity of change of base point (see [11, Sections 2.3.1 and 3.2.5]).

Proposition 3.17. If $\left(\Sigma^{B}, h_{1}, \Sigma_{1}^{B}\right)$ is equivalent to $\left(\Sigma^{B}, h_{2}, \Sigma_{2}^{B}\right)$ in $A_{B} \subset T\left(\Sigma^{B}\right)$, then the corresponding maps $f^{\mu_{1}}$ and $f^{\mu_{2}}$ are equal on $\alpha\left(\partial \Sigma^{B}\right)$. In particular, $\Lambda$ is well defined. 
DAVID RADNELL AND ERIC SCHIPPERS

Proof. Let $\left[\Sigma^{B}, h_{1}, \Sigma_{1}^{B}\right]=\left[\Sigma^{B}, h_{2}, \Sigma_{2}^{B}\right]$, so that there exists a biholomorphism $\gamma$ : $\Sigma_{1}^{B} \rightarrow \Sigma_{2}^{B}$ such that $h_{2}^{-1} \circ \gamma \circ h_{1}$ is homotopic to the identity rel $\partial \Sigma^{B}$. Since the dilatation of $\gamma \circ h_{1}$ and $h_{1}$ are equal, composition by $\gamma$ does not change the resulting $f^{\mu}$ in the definition of $\Lambda$. Thus we may absorb $\gamma$ into $h_{1}$ and assume that $\Sigma_{1}^{B}=\Sigma_{2}^{B}$ and $h_{2}^{-1} \circ h_{1}$ is homotopic to the identity rel $\partial \Sigma^{B}$. In particular, $h_{1}=h_{2}$ on $\partial \Sigma^{B}$ and, moreover, $\tilde{h}_{1}$ is homotopic to $\tilde{h}_{2}$ on $\Sigma^{P}$.

Now let $\sigma: \Sigma^{\mu} \rightarrow \Sigma_{*}^{P}$ be the fixed biholomorphism in the definition of $\Lambda$. Let $f^{\mu_{i}}: \Sigma_{G} \rightarrow \Sigma^{\mu}$ be the pair of maps with dilatations $\mu_{i}=\mu\left(\tilde{h}_{i} \circ \alpha^{-1}\right)$ respectively.

Since $\tilde{h}_{i} \circ \alpha^{-1}$ and $f^{\mu_{i}}$ have the same dilatations for $i=1,2$, there is a pair of biholomorphic maps $\delta_{i}: \Sigma_{i}^{B} \# h_{h_{i} \circ \tau} \overline{\mathbb{D}}_{0}^{n} \rightarrow \Sigma^{\mu}$ such that

$$
\delta_{i} \circ \tilde{h}_{i} \circ \alpha^{-1}=f^{\mu_{i}}
$$

on $\Sigma_{G}$. Because $h_{1}=h_{2}$ on $\partial \Sigma^{B}$ and $\Sigma_{1}^{B}=\Sigma_{2}^{B}, \Sigma_{1}^{B} \#_{h_{1} \circ \tau} \overline{\mathbb{D}}_{0}^{n}=\Sigma_{2}^{B} \#_{h_{2} \circ \tau} \overline{\mathbb{D}}_{0}^{n}$.

By Remark 3.15, we know that $\left(f^{\mu_{2}}\right)^{-1} \circ f^{\mu_{1}}$ is homotopic to the identity on $\Sigma_{G}$. Thus $\delta_{2}^{-1} \circ \delta_{1}$ is homotopic to the identity. Since $\Sigma^{P}$ is of type $(g, n)$ with $2 g-2+n>0$ the biholomorphism $\delta_{2}^{-1} \circ \delta_{1}$ must be the identity. We can conclude that $\delta_{1}=\delta_{2}$. Therefore, since $h_{1}=h_{2}$ on $\partial \Sigma^{B}$, by (3.6) $f^{\mu_{1}}=f^{\mu_{2}}$ on $\alpha\left(\partial \Sigma^{B}\right)$, so

$$
\Lambda\left(\left[\Sigma^{B}, h_{1}, \Sigma_{1}^{B}\right]\right)=\Lambda\left(\left[\Sigma^{B}, h_{2}, \Sigma_{2}^{B}\right]\right) .
$$

Let $\mathcal{P}^{-1}$ denote a locally defined inverse of $\mathcal{P}$ (see (2.2)) in a neighborhood of $\left[\Sigma^{P}, f, \Sigma_{*}^{P}, \phi_{0}\right]$. We need to show that

$$
\Lambda \circ \mathcal{P}^{-1} \circ L=\mathrm{id}
$$

on some neighborhood of $\phi_{0}$. For notational simplicity we work with the case of a single puncture on $\Sigma^{P}$. Let $\left[\Sigma^{B}, h_{0}, \Sigma_{*, 0}^{B}\right]=\mathcal{P}^{-1}\left(\left[\Sigma^{P}, f, \Sigma_{*}^{P}, \phi_{0}\right]\right)$ and recall that $\Sigma_{*, 0}^{B}=\Sigma_{*}^{P} \backslash \phi_{0}(\mathbb{D})$ and $h_{0} \circ \tau=\phi_{0}$ on $\partial \mathbb{D}$.

Choose a compact set $E \subset \Sigma_{*}^{P}$ such that $E \subset \phi_{0}(\mathbb{D})$. Let $V_{0} \subset \mathcal{O}_{\mathrm{qc}}\left(\Sigma_{*}^{P}\right)$ be an open neighborhood of $\phi_{0}$ such that for all $\phi \in V_{0}, E \subset \phi(\mathbb{D})$. This is possible by [14, Corollary 3.4].

Let $\iota_{0}: \Sigma_{*, 0}^{B} \# h_{0} \circ \tau=\overline{\mathbb{D}}_{0} \rightarrow \Sigma_{*}^{P}$ be the biholomorphism defined by

$$
\iota_{0}(z)= \begin{cases}z, & z \in \Sigma_{*, 0}^{B}, \\ \phi_{0}(z), & z \in \mathbb{D} .\end{cases}
$$

Since $h_{0} \circ \tau=\phi_{0}$ on $\partial \mathbb{D}$, we have

$$
\iota_{0} \circ \tilde{h}_{0} \circ \tilde{\tau}=\phi_{0}
$$

Let

$$
\sigma=\iota_{0} \circ \delta_{0}^{-1}: \Sigma^{\mu} \rightarrow \Sigma_{*}^{P}
$$

where $\delta_{0}: \Sigma_{*, 0}^{B} \#_{h_{0} \circ \tau} \overline{\mathbb{D}}_{0} \rightarrow \Sigma^{\mu}$ is the unique biholomorphism satisfying (3.6). Use the map $\sigma$ for the biholomorphism in the definition of $\Lambda$. Now let $\psi=$ $\Lambda\left(\left[\Sigma^{B}, h_{0}, \Sigma_{*, 0}^{B}\right]\right)$. It follows directly that $\psi=\phi_{0}$ :

$$
\begin{aligned}
\psi & =\sigma \circ f^{\mu_{0}} \circ \alpha \circ \tilde{\tau} \\
& =\iota_{0} \circ \delta_{0}^{-1} \circ\left(\delta_{0} \circ \tilde{h}_{0} \circ \alpha^{-1}\right) \circ \alpha \circ \tilde{\tau} \\
& =\iota_{0} \circ \tilde{h}_{0} \circ \tilde{\tau} .
\end{aligned}
$$

Thus by equation (3.8) $\psi=\phi_{0}$. 
For $\phi \in V_{0}$, let $\left[\Sigma_{B}, h_{\phi}, \Sigma_{*, \phi}^{B}\right]=\left(\mathcal{P}^{-1} \circ L\right)(\phi)$ where $\Sigma_{*, \phi}^{B}=\Sigma_{*}^{P} \backslash \phi\left(\mathbb{D}_{0}\right)$. We eventually want to show that for any $\phi \in V_{0}, \Lambda\left(\left[\Sigma_{B}, h_{\phi}, \Sigma_{*, \phi}^{B}\right]\right)=\phi$.

Choose a holomorphic curve $\phi_{t} \in V_{0}$ joining $\phi_{0}$ and $\phi$. That is, $\phi_{1}=\phi$ and $\phi_{0}$ is as above. By Remark 3.12, $\phi_{t}(z)$ is holomorphic in $t$ and so the construction in the proof of Lemma 3.11 can be repeated. Let $\Sigma_{*, t}^{B}=\Sigma_{*, \phi_{t}}^{B}$ and define $F_{t} \circ h_{0}$ : $\Sigma^{B} \rightarrow \Sigma_{*, t}^{B}$ where $F_{t}$ is as in (3.3). As before, $t \mapsto \mu\left(F_{t} \circ h_{0}\right)$ is holomorphic. Let $h_{t}=F_{t} \circ h_{0}, \mu_{t}=\mu\left(\tilde{h}_{t} \circ \alpha^{-1}\right)$, and let $\delta_{t}: \Sigma_{*, t}^{B} \# h_{t} \circ \overline{\mathbb{D}} \rightarrow \Sigma^{\mu}$ be the biholomorphism defined by

$$
\delta_{t}=f^{\mu_{t}} \circ \alpha \circ \tilde{h}_{t}^{-1}
$$

as in (3.6). Define $\iota_{t}$ as in (3.7) by replacing $\phi_{0}$ with $\phi_{t}$.

Lemma 3.18. The map $\delta_{t}: \Sigma_{*, t}^{B} \#_{h_{t} \circ \tau} \overline{\mathbb{D}}_{0} \rightarrow \Sigma^{\mu}$ is holomorphic in $t$ for $z \in \mathbb{D}$.

Proof. Since $t \mapsto \mu\left(h_{t}\right)$ is holomorphic in $t$, Remark 3.16] shows that $\mu_{t}=\mu\left(\tilde{h}_{t} \circ \alpha^{-1}\right)$ is holomorphic in $t$. Thus for fixed $z, w^{\mu_{t}}(z)$ is holomorphic in $t$. Because $\pi^{\mu}$ is independent of $\mu$ by Lemma 3.13 and Proposition 3.14, $f^{\mu_{t}}(z)$ is also holomorphic in $t$ for fixed $z$.

For $z \in \mathbb{D}$,

$$
\delta_{t}(z)=\left(f^{\mu_{t}} \circ \alpha \circ \tilde{h}_{t}^{-1}\right)(z)=\left(f^{\mu_{t}} \circ \alpha\right)(z)
$$

from the definition of $\delta_{t}$, and the conclusion follows immediately.

Lemma 3.19. $\sigma=\iota_{0} \circ \delta_{0}^{-1}=\iota_{t} \circ \delta_{t}^{-1}$.

Proof. Let

$$
\beta_{t}=\sigma \circ \delta_{t} \circ \iota_{t}^{-1}: \Sigma_{*}^{P} \rightarrow \Sigma_{*}^{P} .
$$

We will show that $\beta_{t}$ is the identity. First we claim that $\beta_{t}$ is holomorphic in $t$ for $z \in E$. We have that $\phi_{t}(z)$ is holomorphic as a function of $t$ and $z$ and thus so is $\phi_{t}^{-1}(z)$ by a direct application of the implicit function theorem. For $z \in E$, and using $\iota_{t} \circ \tilde{h}_{t} \circ \tilde{\tau}=\phi_{t}$ from (3.8),

$$
\begin{aligned}
\beta_{t}(z) & =\left(\sigma \circ f^{\mu_{t}} \circ \alpha \circ \tilde{h}_{t}^{-1} \circ \iota_{t}^{-1}\right)(z) \\
& =\left(\sigma \circ f^{\mu_{t}} \circ \alpha \circ \tilde{\tau} \circ \phi_{t}^{-1}\right)(z)
\end{aligned}
$$

which is holomorphic in $t$.

The fact that $\beta_{t}$ is the identity follows from the following four observations. (1) By Hurwitz's theorem, the automorphism group of $\Sigma_{*}^{P}$ is finite. (2) $\beta_{t}$ is the identity if and only if $\left.\beta_{t}\right|_{E}$ is the identity. (3) For $z \in E, \beta_{t}(z)$ is continuous in $t$. (4) From the definition of $\sigma, \beta_{0}$ is the identity.

Theorem 3.20. For all $\phi \in V_{0}$, and any local inverse $\mathcal{P}^{-1}$ of $\mathcal{P},\left(\Lambda \circ \mathcal{P}^{-1} \circ L\right)(\phi)$ $=\phi$.

Proof. We join $\phi$ to $\phi_{0}$ by a holomorphic curve $\phi_{t}$ such that $\phi_{1}=\phi$. From Lemma 3.19 and equation (3.6) we have:

$$
\begin{aligned}
\left(\Lambda \circ \mathcal{P}^{-1} \circ L\right)\left(\phi_{t}\right) & =\Lambda\left(\left[\Sigma^{B}, h_{t}, \Sigma_{*, 0}^{B}\right]\right) \\
& =\sigma \circ f^{\mu_{t}} \circ \alpha \circ \tilde{\tau} \\
& =\left(\iota_{t} \circ \delta_{t}^{-1}\right) \circ\left(\delta_{t} \circ \tilde{h}_{t} \circ \alpha^{-1}\right) \circ \alpha \circ \tilde{\tau} \\
& =\iota_{t} \circ \tilde{h}_{t} \circ \tilde{\tau} \\
& =\phi_{t},
\end{aligned}
$$


where the last equality follows as both $\tilde{\tau}$ and $\tilde{h}_{t}$ are the identity on $\mathbb{D}$. Setting $t=1$ completes the proof.

\section{5. $L^{-1}$ is holomorphic.}

Lemma 3.21. Let $\left[\Sigma^{B}, h_{0}, \Sigma_{*, 0}^{B}\right]=\mathcal{P}^{-1}\left(\left[\Sigma^{P}, f, \Sigma_{*}^{P}, \phi_{0}\right]\right)$ for some local inverse $\mathcal{P}^{-1}$ of $\mathcal{P}$. Let $B$ be an open set in $\Sigma_{*}^{P}$ such that $\left(\sigma \circ f^{\mu_{0}} \circ \alpha \circ \tilde{\tau}\right)\left(\overline{\mathbb{D}}_{0}\right) \subset B$. There exists a neighborhood $A_{0} \subset F_{B}$ of $\left[\Sigma^{B}, h_{0}, \Sigma_{*, 0}^{B}\right]$ such that

$$
\Lambda\left(\left[\Sigma^{B}, h, \Sigma_{1}^{B}\right]\right)\left(\overline{\mathbb{D}}_{0}\right) \subset B
$$

for all $\left[\Sigma^{B}, h, \Sigma_{1}^{B}\right] \in A_{0}$.

Proof. Let $\gamma$ be a local holomorphic section of the fundamental projection $\Phi$ in a neighborhood of $\left[\Sigma^{B}, h_{0}, \Sigma_{*, 0}^{B}\right]$, and choose $U$ to be an open set in the domain of $\gamma$ containing this point. Given $u \in U$ we choose a representative $\left[\Sigma^{B}, h_{u}, \Sigma_{1}^{B}\right]=u$ such that $\mu\left(h_{u}\right)=\gamma(u)$.

By Remark 3.16, $\mu\left(h_{u}\right) \mapsto \mu\left(\tilde{h}_{u} \circ \alpha^{-1}\right)$ is holomorphic. Therefore, since $\gamma$ is holomorphic, the map

$$
\begin{aligned}
U & \rightarrow L_{-1,1}^{\infty}\left(\Sigma_{G}\right) \\
u & \mapsto \mu\left(\tilde{h}_{u} \circ \alpha^{-1}\right)
\end{aligned}
$$

is holomorphic and in particular continuous.

We now show in general that $w^{\mu}(z)$ and hence $f^{\mu}(z)$ are jointly continuous in $\mu$ and $z$. Let $\mathbb{D}_{r}=\{z:|z|<r\}$ for some $r>1$. Fix $\mu_{0}$ and $z_{0}$. By [5, Theorem 4.7.4], for any $\epsilon>0$ there is a $\delta_{1}$ so that if $\left\|\mu-\mu_{0}\right\|<\delta_{1}$, then $\left|w_{\mu}(z)-w_{\mu_{0}}(z)\right|<\epsilon / 2$ for all $z \in \overline{\mathbb{D}}_{r}$. On the other hand, since $w_{\mu_{0}}$ is continuous in $z$ there is a $\delta_{2}$ such that $\left|w_{\mu_{0}}(z)-w_{\mu_{0}}\left(z_{0}\right)\right|<\epsilon / 2$ for all $\left|z-z_{0}\right|<\delta_{2}$. We can assume that $\delta_{2}$ is small enough that the disk of radius $\delta_{2}$ centered on $z_{0}$ is contained in $\mathbb{D}_{r}$. Thus for $\left|z-z_{0}\right|<\delta_{2}$ and $\left\|\mu-\mu_{0}\right\|<\delta_{1}$,

$$
\left|w_{\mu}(z)-w_{\mu_{0}}\left(z_{0}\right)\right| \leq\left|w_{\mu}(z)-w_{\mu_{0}}(z)\right|+\left|w_{\mu_{0}}(z)-w_{\mu_{0}}\left(z_{0}\right)\right|<\epsilon .
$$

So $w^{\mu}(z)$ is jointly continuous in $\mu$ and $z$ for all $z \in \mathbb{D}$ and hence so is $f^{\mu}(z)$.

By applying this fact with $\mu=\mu\left(\tilde{h} \circ \alpha^{-1}\right)$, we have that

$$
(u, z) \mapsto f^{\mu}(z)
$$

is continuous.

In the following let $D(\zeta, R)$ denote the disc of radius $R$ centered on $\zeta$. Let $B$ be as in Section 3.1 (recall that we are assuming that there is a single puncture). Since each $\left(\sigma \circ f^{\mu} \circ \alpha\right)\left(\partial \Sigma^{B}\right)$ is compact, there is an $r$ so that $D(\xi, r) \subset B$ for all $\xi \in\left(\sigma \circ f^{\mu} \circ \alpha\right)\left(\partial \Sigma^{B}\right)$. By the continuity of $f^{\mu}(\zeta)$ in both $\mu$ and $\zeta$ for each $\zeta \in \alpha\left(\partial \Sigma^{B}\right)$, and by letting $\xi=\left(\sigma \circ f^{\mu}\right)(z)$, one can choose an open neighborhood $U_{\xi}=D\left(z, \delta_{\xi}\right) \times W_{\xi}$ of $\left(z,\left[\Sigma^{B}, h_{0}, \Sigma_{*, 0}^{B}\right]\right)$ so that $f^{\mu}(\zeta) \subset B(\xi, r)$ for all $\zeta \in D\left(z, \delta_{\xi}\right)$ and $\left[\Sigma^{B}, h_{u}, \Sigma_{1}^{B}\right] \in W_{\xi}$. Since $\alpha\left(\partial \Sigma^{B}\right)$ is compact, its open cover by the union of $D\left(z, \delta_{\xi}\right)$ has a finite subcovering $D\left(z_{1}, \delta_{\xi_{1}}\right), \ldots, D\left(z_{m}, \delta_{\xi_{m}}\right)$. The open set $A_{0}=$ $W_{\xi_{1}} \cap \cdots \cap W_{\xi_{m}}$ has the desired properties.

Fix a point $z_{0} \in \alpha\left(\mathbb{D}_{0}\right)$. Choose $Q \subset \mathbb{D}_{0}$ to be an open neighborhood of $z_{0}$ such that there exists a local holomorphic section $s$ of $\pi_{G}: U \rightarrow U / G=\Sigma_{G}$ defined on 
$(\alpha \circ \tau)(Q)$. Let $\pi=\pi^{\mu}$, which is independent of $\mu$ by Lemma 3.13 and Proposition 3.14. For $z \in Q$, we can now write $\Lambda$ in terms of $w^{\mu}$ and fixed maps as follows:

$$
\Lambda\left(\left[\Sigma^{B}, h, \Sigma_{1}^{B}\right]\right)=\sigma \circ\left(\pi \circ w^{\mu} \circ s\right) \circ \alpha \circ \tilde{\tau} .
$$

With the aid of Theorem 3.8 we can now proceed with the proof that $\Lambda$ is holomorphic.

Lemma 3.22. Let $t \mapsto\left[\Sigma^{B}, h_{t}, \Sigma_{t}^{B}\right]$ be a holomorphic curve in $A_{B}$. For any $z \in \mathbb{D}$, $\phi_{t}(z)=\Lambda\left(\left[\Sigma^{B}, h_{t}, \Sigma_{t}^{B}\right]\right)(z)$ and all its derivatives in $z$ are holomorphic in $t$.

Proof. We first show that the claim holds for a neighborhood of any fixed $z_{0}$ in $\mathbb{D}_{0}$. By the existence of holomorphic sections of the fundamental projection the curve $t \mapsto\left[\Sigma^{B}, h_{t}, \Sigma_{t}^{B}\right]$ is the image of a holomorphic curve in $L_{-1,1}^{\infty}\left(\Sigma^{B}\right)_{1}$. We can thus assume without loss of generality that our representatives $\left(\Sigma^{B}, h_{t}, \Sigma_{t}^{B}\right)$ are such that $t \mapsto \mu\left(h_{t}\right)$ is holomorphic in $t$. Thus the maps $t \mapsto \mu_{t}=\mu\left(\tilde{h}_{t}\right)$ and $t \mapsto w^{\mu_{t}}(z)$ are holomorphic in $t$ as in the proof of Lemma 3.18 .

Let $C=\alpha\left(\mathbb{D}_{0}\right)$ be the cap on $\Sigma_{G}$. Since $\mu_{t}$ is zero on $C, w^{\mu_{t}}(z)$ is holomorphic in $z$ for $z \in s(C)$. Therefore, it is a holomorphic function of $t$ and $z$ and hence all its derivatives are also holomorphic functions of both variables. The statement for $\phi_{t}$ then follows from (3.9) for all $z$ in some neighborhood $Q$ of $z_{0}$. This proves the claim for $z \neq 0$.

To prove the claim for $z_{0}=0$, observe that for all $t, \phi_{t}(0)=p$. So in fact we know that $\phi_{t}(z)$ is holomorphic in $z$ for fixed $t$ for all $z \in \mathbb{D}$. Furthermore, clearly $\phi_{t}(0)$ is holomorphic in $t$. Thus $\phi$ is holomorphic in $t$ and $z$ separately and so by Hartog's theorem $\phi$ is jointly holomorphic in both $t$ and $z$. Thus all the derivatives of $\phi$ with respect to $z$ are holomorphic in $t$ for any fixed $z \in \mathbb{D}$.

Fix the point $\left[\Sigma^{B}, h_{0}, \Sigma_{*, 0}^{B}\right] \in F_{B}$, and let $B_{i}, i=1, \ldots, n$ and $A_{0}$ be as in Lemma 3.21] it is possible to choose the $B_{i}$ to be non-overlapping. Let $\zeta_{i}: B_{i} \rightarrow \mathbb{D}$, $i=1, \ldots, n$ be biholomorphisms. From Remark 3.1 there is a corresponding chart $T: \mathcal{O}_{\mathrm{qc}}\left(\Sigma_{*}^{P}\right) \rightarrow \mathcal{O}_{\mathrm{qc}}^{n}$ on the open subset $V=\left\{\phi \in \mathcal{O}_{\mathrm{qc}}\left(\Sigma_{*}^{P}\right) \mid \phi_{i}(\overline{\mathbb{D}}) \subset B_{i}\right\}$ containing $\Lambda\left(\left[\Sigma^{B}, h_{0}, \Sigma_{0}^{B}\right]\right)$, given by $T\left(\left(\phi_{1}, \ldots, \phi_{n}\right)\right)=\left(\zeta_{1} \circ \phi_{1}, \ldots, \zeta_{n} \circ \phi_{n}\right)$.

Theorem 3.23. There exists an open neighborhood $A_{0}$ of $\left[\Sigma^{B}, h_{0}, \Sigma_{*, 0}^{B}\right] \in A_{B}$ such that $\Lambda: A_{0} \rightarrow \mathcal{O}_{\mathrm{qc}}\left(\Sigma_{*}^{P}\right)$ is holomorphic.

Proof. Choose $A_{0}$ as in Lemma 3.21 and the preceding paragraph.

Using Theorems 3.7 and 3.8 it is enough to show weak Gâteaux-holomorphy and local boundedness. As before we temporarily drop the subscript $i$ for ease of notation.

Let $\phi_{t}$ be as in Lemma 3.22 where it was proved that $t \mapsto \phi_{t}(z)$ is holomorphic in $t$. The complex structure on $\mathcal{O}_{\mathrm{qc}}\left(\Sigma_{*}^{P}\right)$ is defined by

$$
\mathcal{O}_{\mathrm{qc}}\left(\Sigma_{*}^{P}\right) \stackrel{T}{\longrightarrow} \mathcal{O}_{\mathrm{qc}} \stackrel{\chi}{\longrightarrow} A_{\infty}^{1} \oplus \mathbb{C} .
$$

Recall from Section 3.1 that $\chi(f)=\left(\mathcal{A}(f), f^{\prime}(0)\right)$ where $\mathcal{A}(f)=f^{\prime \prime} / f^{\prime}$. Let $\psi_{t}=$ $T\left(\phi_{t}\right)=\zeta \circ \phi_{t}$. We now need to prove that $t \mapsto \chi\left(T\left(\phi_{t}\right)\right)$ satisfies condition (1) of Theorem 3.8 .

This is immediate for the second component of $\chi$ because $\phi_{t}(0)$ is holomorphic in $t$. Since $\phi_{t}(z)$ and its derivatives are holomorphic in $t$ for fixed $z$, we see that

$$
t \mapsto\left(\mathcal{A}\left(\psi_{t}\right)\right)(z)
$$


is also holomorphic in $t$. Define $E_{z}: A_{\infty}^{1} \rightarrow \mathbb{C}$ by $E_{z}(f)=f(z)$. These point evaluation maps are continuous linear functionals for $z \in \mathbb{D}$. If $\Omega$ is an open subset of $\mathbb{D}$, then $\left\{E_{z} \mid z \in \Omega\right\}$ is a separating set of functionals because the holomorphic functions are determined by their values on an open set.

By Theorem 3.8 it remains to show that $\Lambda$ is locally bounded. From the definition of the complex structure on $\mathcal{O}_{\mathrm{qc}}\left(\Sigma_{*}^{P}\right)$ we must prove that $\chi \circ T \circ \Lambda$ is locally bounded.

It suffices to show that each component of $\chi \circ T \circ \Lambda$ in $A_{\infty}^{1} \oplus \mathbb{C}$ is bounded uniformly on $A_{0}$. For any element of $A_{0}$ the corresponding map

$$
\psi=\zeta \circ \sigma \circ f^{\mu} \circ \alpha \circ \tilde{\tau} \in \mathcal{O}_{\mathrm{qc}}
$$

is a holomorphic map from $\mathbb{D}$ to $\mathbb{D}$ satisfying $\psi(0)=0$. By the Schwarz lemma

$$
\left|\psi^{\prime}(0)\right| \leq 1 \text {. }
$$

Furthermore, by an elementary estimate for univalent maps of the disk (using again the above bound on the first derivative),

$$
\left|\left(1-|z|^{2}\right) \mathcal{A}(\psi)(z)-2 \bar{z}\right| \leq 4,
$$

and so

$$
\|\mathcal{A}(\psi)\|_{1, \infty} \leq 6
$$

By Theorem 3.20 and the preceding theorem we now conclude that $\Lambda \circ \mathcal{P}^{-1}$ is a local holomorphic inverse of $L$.

\section{Coordinates on the Teichmüller space $T\left(\Sigma^{B}\right)$}

Schiffer variation together with coordinates on the fibers $F_{B}$ provide local holomorphic coordinate charts for the infinite-dimensional Teichmüller space $T\left(\Sigma^{B}\right)$.

Let $S: \Omega \rightarrow T\left(\Sigma^{P}\right)$ be the coordinates in a neighborhood of $\left[\Sigma^{P}, f, \Sigma_{*}^{P}\right] \subset T\left(\Sigma^{P}\right)$ obtained by Schiffer variation as described in Theorem 2.17. Let $\Sigma_{\epsilon}^{P}=\left(\Sigma_{*}^{P}\right)^{\epsilon}$ and recall that $f^{\epsilon}=\nu^{\epsilon} \circ f$.

Note in particular that for any $\epsilon \neq 0$ in $\Omega, S(\epsilon) \neq\left[\Sigma^{P}, f, \Sigma_{*}^{P}\right]$. This implies that Schiffer variation in $\Sigma^{B}$ is transverse to the fibers $F_{B}$.

Let $(T, V)$ be a chart on $\mathcal{O}_{\mathrm{qc}}\left(\Sigma_{*}^{P}\right)$ as in Section 3.1. Recall that this chart can be chosen so that for all $\phi \in V, \phi\left(\overline{\mathbb{D}}_{0}\right)$ is contained in some fixed open $B \subset \Sigma_{*}^{P}$. Now choose the neighborhoods $U_{i}, i=1, \ldots, d$, on which the Schiffer variation is to be performed, to be disjoint from $B$.

Let $f_{\phi}$ be as in Corollary 3.3 .

Theorem 4.1. The map

$$
\begin{aligned}
(\Omega \times V) & \rightarrow T\left(\Sigma^{B}\right) \\
(\epsilon, \phi) & \mapsto\left[\Sigma^{B},\left(f_{\phi}\right)^{\epsilon},\left(\Sigma_{*}^{P} \backslash \phi\left(\mathbb{D}_{0}^{n}\right)\right)^{\epsilon}\right]
\end{aligned}
$$

is biholomorphic onto its image.

Proof. Because $\mathcal{P}$ is a local biholomorphism, it is sufficient that

$$
\begin{aligned}
G:(\Omega \times V) & \rightarrow \widetilde{T}^{P}\left(\Sigma^{P}\right) \\
(\epsilon, \phi) & \mapsto\left[\Sigma^{P}, \nu^{\epsilon} \circ f, \Sigma_{\epsilon}^{P}, \nu^{\epsilon} \circ \phi\right]
\end{aligned}
$$

is a biholomorphism onto its image. That $G$ is injective follows directly from the definition of $\widetilde{T}^{P}\left(\Sigma^{P}\right)$ and the facts: (1) $S: \Omega \rightarrow T\left(\Sigma^{P}\right)$ is injective, and (2) 
$\Sigma^{P}$ has no non-identity automorphisms that are homotopic to the identity since $2 g-2+n>0$.

First, we show that this map is holomorphic. By Hartog's theorem, it is enough to show that the map is separately holomorphic (see [10 for a version of this theorem in infinite dimensions). Fixing $\phi$, by Theorem 2.18 the map $\epsilon \mapsto\left[\Sigma^{B}, f_{\phi}^{\epsilon},\left(\Sigma_{*}^{P} \backslash\right.\right.$ $\left.\left.\phi\left(\mathbb{D}_{0}^{n}\right)\right)^{\epsilon}\right]$ is holomorphic. Thus since $\mathcal{P}$ is holomorphic, $G$ is holomorphic in $\epsilon$. Now fix $\epsilon$, and consider the map corresponding to the second component of $G$ :

$$
\begin{aligned}
H: V & \rightarrow \widetilde{T}^{P}\left(\Sigma^{P}\right) \\
\phi & \mapsto\left[\Sigma^{P}, \nu^{\epsilon} \circ f, \Sigma_{\epsilon}^{P}, \nu^{\epsilon} \circ \phi\right] .
\end{aligned}
$$

Now $H$ can be written as $H_{2} \circ H_{1}$ where $H_{1}$ and $H_{2}$ are given by

$$
\begin{aligned}
H_{1}: V & \rightarrow \mathcal{O}_{\mathrm{qc}}\left(\Sigma_{\epsilon}^{P}\right) \\
\phi & \mapsto \nu^{\epsilon} \circ \phi
\end{aligned}
$$

and

$$
\begin{aligned}
H_{2}: \mathcal{O}_{\mathrm{qc}}\left(\Sigma_{\epsilon}^{P}\right) & \rightarrow \widetilde{T}^{P}\left(\Sigma^{P}\right) \\
\xi & \mapsto\left[\Sigma^{P}, \nu^{\epsilon} \circ f, \Sigma_{\epsilon}^{P}, \xi\right] .
\end{aligned}
$$

$H_{2}$ is holomorphic by Theorem 3.2, so it remains to show that $H_{1}$ is holomorphic.

Let $\zeta$ be the collection of local biholomorphisms of neighborhoods of the punctures on $\Sigma_{*}^{P}$ corresponding to the chart $(T, V)$ on $\mathcal{O}_{\mathrm{qc}}\left(\Sigma_{*}^{P}\right)$. Let $\left(T_{\epsilon}, V_{\epsilon}\right)$ be the chart on $\mathcal{O}_{\mathrm{qc}}\left(\Sigma_{\epsilon}^{P}\right)$ and $\zeta_{\epsilon}$ be the corresponding local biholomorphism of $\Sigma_{\epsilon}^{P}$. We need to show that $T_{\epsilon} \circ H_{1} \circ T^{-1}$ is holomorphic, i.e., that the map

$$
\zeta \circ \phi \mapsto \zeta_{\epsilon} \circ \nu_{\epsilon} \circ \phi=\left(\zeta_{\epsilon} \circ \nu_{\epsilon} \circ \zeta^{-1}\right) \circ(\zeta \circ \phi)
$$

is holomorphic on $\mathcal{O}_{\mathrm{qc}}$. Composition on the left by a biholomorphism is a local biholomorphism of $\mathcal{O}_{\mathrm{qc}}$ by [14, Lemma 3.10], which establishes the claim.

Finally we need to show that $G^{-1}$ is holomorphic. By Theorem 3.2 and the fact that Schiffer variation provides a section of the fiber projection, it is clear that the derivative of $G$ is an injective linear map at each point for which $G$ is defined. Since $G$ is holomorphic and in particular $C^{1}$, we can apply the inverse function theorem [6] to show that $G$ has a $C^{1}$ inverse. The derivative of $G^{-1}$ is also complex linear so $G^{-1}$ is holomorphic.

\section{REFERENCES}

1. Ahlfors, L. V. and Sario, L., Riemann surfaces, Princeton Mathematical Series, no. 26, Princeton University Press, Princeton, NJ, 1960. MR0114911 (22:5729)

2. Chae, S. B., Holomorphy and calculus in normed spaces. With an appendix by Angus E. Taylor. Monographs and Textbooks in Pure and Applied Mathematics, 92. Marcel Dekker, Inc., New York, 1985. MR788158 (86j:46044)

3. Gardiner, F. P., Schiffer's interior variation and quasiconformal mapping. Duke Math. J. 42 (1975), 371-380. MR0382637 (52:3519)

4. Grosse-Erdmann, K.-G., A weak criterion for vector-valued holomorphy, Math. Proc. Cambridge Philos. Soc., 169 (2004), no. 2, 399-411. MR 2040581 (2005a:30079)

5. Hubbard, J. H., Teichmüller theory and applications to geometry, topology, and dynamics. Vol. 1., Matrix Editions, Ithaca, New York, 2006. MR.2245223 (2008k:30055)

6. Lang, S., Differential manifolds. Second edition. Springer-Verlag, New York, 1985. MR772023 (85m:58001)

7. Lehto, O., Quasiconformal mappings and singular integrals. Instituto Nazionale di Alta Matematica., Symposia Mathematica XVIII 429-453, Academic Press 1976. MR0492241 $(58: 11387)$ 
8. Lehto, O., Univalent functions and Teichmüller spaces. Graduate Texts in Mathematics 109 Springer-Verlag, New York, 1987. MR867407 (88f:30073)

9. Lehto, O. and Virtanen, K. I., Quasiconformal mappings in the plane. 2nd edition. Die Grundlehren der mathematischen Wissenshcaften, Band 126. Springer-Verlag, New YorkHeidelberg, 1973. MR0344463 (49:9202)

10. Mujica, J., Complex Analysis in Banach Spaces. North Holland, 1986. MR842435 (88d:46084)

11. Nag, S., The complex analytic theory of Teichmüller spaces. Canadian Mathematical Society Series of Monographs and Advanced Texts. A Wiley-Interscience Publication. John Wiley \& Sons, Inc., New York, 1988. MR.927291 (89f:32040)

12. Radnell, D., Schiffer Variation in Teichmüller space, Determinant Line Bundles and Modular Functors, PhD Thesis, Rutgers University, New Brunswick, NJ, 2003.

13. Radnell, D. and Schippers, E., Quasisymmetric sewing in rigged Teichmüller space, Commun. Contemp. Math. 8 (2006) no. 4, 481-534. arXiv:math-ph/0507031. MR.2258875 (2007f:32015)

14. Radnell, D. and Schippers, E., A complex structure on the set of quasiconformally extendible non-overlapping maps into a Riemann surface, J. Anal. Math. 108 (2009) no. 1, 277-291. arXiv:0803.3211. MR2544761

15. Radnell, D. and Schippers, E., The semigroup of rigged annuli and the Teichmüller space of the annulus. Preprint, 2009. MR.2544761

16. Segal, G., The definition of conformal field theory, Topology, Geometry and Quantum Field Theory (U. Tillmann, ed.), London Mathematical Society Lecture Note Series, vol. 308, Cambridge University Press, 2004; original preprint 1988, pp. 421-576. MR2079383(2005h:81334)

17. Teo, L.-P., Velling-Kirillov metric on the universal Teichmüller curve. J. Anal. Math. 93 (2004), 271-307. MR2110331 (2005k:32014)

18. Takhtajan, L. A. and Teo, L-P., Weil-Petersson metric on the universal Teichmüller space. Mem. Amer. Math. Soc., (2006). MR2251887 (2007e:32011)

19. Słodkowski, Z., Holomorphic motions and polynomial hulls, Proc. Amer. Math. Soc., 111(2), (1991), 347-355. MR 1037218(91f:58078)

Department of Mathematics and Statistics, American University of Sharjah, P.O. Box 26666, Sharjah, United Arab Emirates

E-mail address: dradnell@aus.edu

Department of Mathematics, University of Manitoba, Winnipeg, MB, R3T 2N2, CANADA

E-mail address: eric_schippers@umanitoba.ca 